\title{
Self-supervising Action Recognition by Statistical Moment and Subspace Descriptors
}

\author{
Lei Wang, Piotr Koniusz * \\ Australian National University and Data61/CSIRO \\ Canberra, Australia \\ \{lei.w,piotr.koniusz\}@anu.edu.au
}

\begin{abstract}
In this paper, we build on a concept of self-supervision by taking RGB frames as input to learn to predict both action concepts and auxiliary descriptors $e$.g., object descriptors. So-called hallucination streams are trained to predict auxiliary cues, simultaneously fed into classification layers, and then hallucinated at the testing stage to aid network. We design and hallucinate two descriptors, one leveraging four popular object detectors applied to training videos, and the other leveraging image- and video-level saliency detectors. The first descriptor encodes the detector- and ImageNet-wise class prediction scores, confidence scores, and spatial locations of bounding boxes and frame indexes to capture the spatio-temporal distribution of features per video. Another descriptor encodes spatio-angular gradient distributions of saliency maps and intensity patterns. Inspired by the characteristic function of the probability distribution, we capture four statistical moments on the above intermediate descriptors. As numbers of coefficients in the mean, covariance, coskewness and cokurtotsis grow linearly, quadratically, cubically and quartically w.r.t. the dimension of feature vectors, we describe the covariance matrix by its leading $n^{\prime}$ eigenvectors (so-called subspace) and we capture skewness/kurtosis rather than costly coskewness/cokurtosis. We obtain state of the art on five popular datasets such as Charades and EPIC-Kitchens.
\end{abstract}

\section{Introduction}

Action Recognition (AR) has progressed from hand-crafted video representations [13, 68, 37, 79, 80, 81, 83, 84] to Convolutional Neural Networks (CNN) [70, 75, 19, 5]. The two-stream networks [70], 3D spatio-temporal features [75], spatio-temporal ResNet model [19] and the new Inflated 3D (I3D) convolutions network pre-trained on Kinetics-400 [5]. Often, AR combine the RGB and optical flow inputs, and benefit from a late fusion (next to the classifier) with low-level representations such as Improved Dense Trajectory (IDT) descriptors [81] due to their highly complementary nature [22, 8, 9, 82, 10]. Recently, AssembleNet and AssembleNet++ [67], learnt with the Neural Architecture Search (NAS) have yielded superb results.

A recent AR pipeline [85], called DEEP-HAL, used IDT descriptors encoded with Bag-of-Words (BoW) [71, 12] and Fisher Vectors (FV) [57, 58] to learn them by so-called hallucination streams and generate at the testing stage to boost results beyond a naive fusion of modalities. DEEP-HAL and approach [74] have shown that even optical flow frames encoded by a network can be learnt by another network trained on RGB frames only, thus pointing at redundancy in training both RGB and optical flow network streams. DEEP-HAL [85] has attained the state of the art on several AR benchmarks by learning to hallucinate IDT-based BoW/FV and Optical Flow Features (OFF) from a single RGB-based I3D network stream.

${ }^{*}$ The corresponding author. This paper is published at the ACM MM'21. DOI: https://doi.org/10. 1145/3474085.3475572. The code will be available at http://users.cecs . anu.edu . au/ koniusz/ 


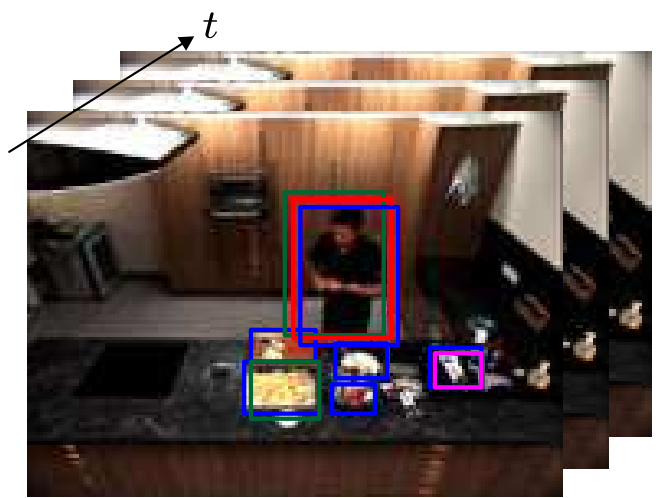

(a)
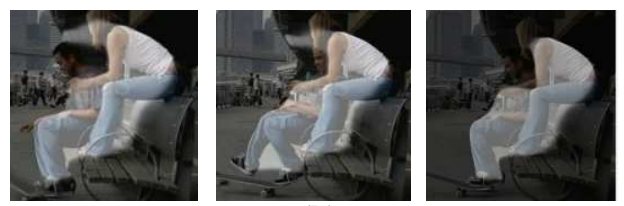

(b)
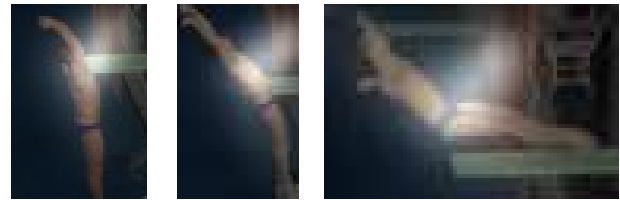

(c)

Figure 1: We use detectors/saliency in hallucination descriptors. Figure 1a shows bounding boxes from four detectors. The faster R-CNN detector with ResNet101 focuses on human-centric actions such as stand, watch, talk, etc. The other three detectors discover e.g., oven, sink, clock, etc. Figure 1b shows that the MNL saliency detector focuses on spatial regions. Figure 1c shows ACLNet saliency detector discovers motion regions.

DEEP-HAL opens up an exciting opportunity to investigate what other representations can coregularize/self-supervise a backbone network for AR with the goal of learning to hallucinate costly representations at the training stage and simply leveraging outputs of halluciantion streams at the testing time. We build on DEEP-HAL which already includes IDT-based BoW/FV and OFF streams. However, we investigate the self-supervisory ability of object/saliency detectors in DEEP-HAL. Moreover, beyond I3D backbone, we investigate the use of AssembleNet and AssembleNet++ but we disable their (impractical to obtain) segmentation mask input.

In this paper, we design and hallucinate two kinds of descriptors, namely Object Detection Features (ODF) and Saliency Detection Features (SDF). The ODF descriptor leverages faster R-CNN detector [62] based on backbones such as (i) Inception V2 [73], (ii) Inception ResNet V2 [72], (iii) ResNet101 [30] and (iv) NASNet [97]. The Inception V2, Inception ResNet V2 and NASNet are pre-trained on the COCO dataset [51] (91 object classes), whereas the ResNet101 is pre-trained on the AVA v2.1 dataset [27] ( 80 human AR classes). The above detectors are applied to training videos to identify humans and objects. Such detected objects together with their relevance and class labels summarized with our descriptor encourage the AR pipeline to focus on semantically important regions and actors relevant to the task of action recognition. Figure 1a shows a few of bounding boxes detected by these four detectors.

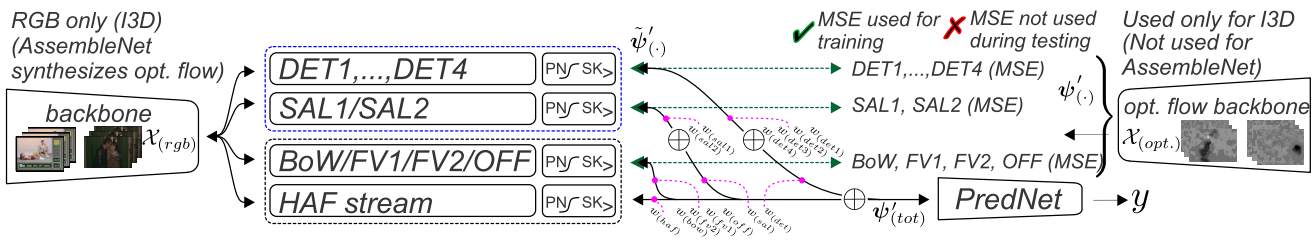

Figure 2: We build on DEEP-HAL [85] which includes I3D RGB and Optical Flow networks (the latter net. is used only during training). For AssembleNet and AssembleNet++, the backbone encodes both RGB and the optical flow, which is synthesized on the fly from RGB frames. For the I3D variant, we remove the prediction and the last 1D conv. layers from I3D RGB and optical flow streams, we feed the $1024 \times 7$ feature representations $\mathcal{X}_{(r g b)}$ into Bag-of-Words $(B o W)$, Fisher Vector $(F V)$, the Optical Flow Features $(O F F)$ and the High Abstraction Features (HAF) streams (dashed black) followed by the Power Normalization (PN) and Sketching $(S K)$ blocks. The OFF stream is supervised by $\mathcal{X}_{(o p t .)}$. For the AssembleNet variant, we obtain the 2048 feature representations $\mathcal{X}_{(r g b)}$ and do not use the OFF stream/optical flow backbone. Moreover, we introduce $D E T 1, \ldots, D E T 4, S A L 1$ and SAL2 streams corresponding to our detector- and saliency-based descriptors (dashed blue). The resulting feature vectors $\tilde{\boldsymbol{\psi}}_{(\cdot)}^{\prime}$, where $(\cdot)$ denotes the stream name $e . g .,(\operatorname{det} 1), \ldots,(\operatorname{det} 2)$ etc., are reweighted by corresponding weights $w_{(\cdot)}$ (magenta lines) and aggregated (sum) by ( $\oplus$ ). All $\tilde{\psi}_{(\cdot)}$ are reweighted, aggregated (sum) and fed to Prediction Network (PredNet). By $\checkmark$, we indicate that the Mean Square Error (MSE) losses are used during training to supervise all streams outputting $\tilde{\boldsymbol{\psi}}_{(\cdot)}^{\prime}$ by the ground-truth $\boldsymbol{\psi}_{(\cdot)}^{\prime}$. By $\boldsymbol{X}$, we indicate that the MSE losses are switched off for testing and $\tilde{\boldsymbol{\psi}}_{(\cdot)}^{\prime}$ are hallucinated/fed into PredNet to obtain labels $y$. 
The SDF leverages image- and video-level saliency detectors such as MNL [95] and ACLNet [94] with the goal of identifying salient regions correlating with the human gaze in spatial and temporal sense. Saliency maps extracted from training videos and summarized by our descriptor help the AR pipeline learn spatial and temporal regions correlating with actions. Figures $1 \mathrm{~b}$ and $1 \mathrm{c}$ show saliency maps from region-wise and temporal saliency detectors.

IDT descriptors are fused with the majority of modern AR pipelines [22, 8, 9, 82, 10] at the classifier level for the best performance while DEEP-HAL [85] learns to hallucinate, and feeds them into the classification branch called PredNet. In this paper, we go further and prepare two compact descriptors, ODF and SDF, and hallucinate them within DEEP-HAL. We equip each hallucination branch with a weighting mechanism adjusted per epoch to attain the best results. Figure 2 illustrates DEEP-HAL at the conceptual level.

For ODF descriptors, we concatenate together per bounding box per frame (i) the one-hot detection and (ii) ImageNet [65] scores, (iii) embedded confidence scores, (iv) embedded bounding box coordinates, and (v) embedded normalized frame index. For all bounding boxes, we stack such features into a matrix. Inspired by the characteristic function of the probability density fun., we extract the mean, leading eigenvectors of covariance, skewness and kurtosis. For SDF descriptors, per frame, we encode saliency via (i) kernelized descriptor on spatio-angular gradient distributions of saliency maps and (ii) intensity patterns. We obtain an ODF per detector and an SDF per saliency detector. Our contributions are as follows: /

i. We propose to use the object and human detectors to enhance the performance of AR pipelines.

ii. We design two types of statistically motivated high-order compact descriptors, Object Detection Features and Saliency Detection Features, for the use in AR pipelines.

iii. We build on the recent DEEP-HAL pipeline [85] but we introduce AssembleNet and AssembleNet++ apart from I3D backbone. Moreover, we introduce a weight learning mechanism for hallucinated feature vectors, and ODF and SDF are hallucinated which leads to the state-of-theart performance.

\section{Related Work}

Below, we describe handcrafted spatio-temporal video descriptors, their encoding strategies and the optical flow used by DEEP-HAL [85]. We also describe deep learning pipelines for video classification. Finally, we discuss the object category and human detectors followed by the spatial and temporal saliency detectors used by us.

Early video descriptors. Early AR used on spatio-temporal interest point detectors [48, 15, 6, 89, 49, 79] and spatio-temporal descriptors [13, 68, 76, 79, 80, 81] which capture various appearance and motion statistics. As spatio-temporal interest point detectors are unable to capture long-term motion patterns, a Dense Trajectory (DT) [79] approach densely samples feature points in each frame to track them in the video (via optical flow). Then, multiple descriptors are extracted along trajectories to capture shape, appearance and motion cues. As DT cannot compensate for the camera motion, the IDT [81, 80] estimates the camera motion to remove the global background motion. IDT also removes inconsistent matches via a human detector. For spatio-temporal descriptors, IDT employs HOG [23], HOF [13] and MBH [80]. HOG [23] contains statistics of the amplitude of image gradients w.r.t. the gradient orientation, thus it captures the static appearance cues. In contrast, HOF [13] captures histograms of optical flow while MBH [80] captures derivatives of the optical flow, thus it is highly resilient to the global camera motion whose cues cancel out due to derivatives. Thus, HOF and MBH contain the zero- and first-order optical flow statistics. Other spatio-temporal descriptors include HOG-3D [37], SIFT3D [68], SURF3D [89] and LTP [92].

We use the DEEP-HAL [85] setup. We encode HOG, HOF, and MBH descriptors on the Improved Dense Trajectories [79, 8, 10] via BoW [71, 12] and FV [57, 58].

BoW/FV encoding. BoW [71, 12] uses a k-means vocabulary to which local descriptors are assigned. Variants include Soft Assignment (SA) [77, 39] and Localized Soft Assignment (LcSA) [52, 43]. As we use DEEP-HAL [85], we use BoW [12] with Power Normalization [43], and FV $[57,58]$ which capture first- and second-order statistics of local descriptors assigned to GMM clusters. DEEP-HAL [85] setup describes how to obtain the BoW/FV global descriptors. 
Optical flow. Older optical flow methods cope with small displacements [31,56] while newer methods cope with larger displacements e.g., Large Displacement Optical Flow (LDOF) [4]. Recent methods use non-rigid descriptor or segment matching [88, 3], or edge-preserving interpolation [63]. We use LDOF [56].

Object detectors. Modern deep learning methods include Region-based Convolutional Neural Networks (R-CNN) [26], its faster variants [25, 62], its mask-based variants [29], and YOLO [61], YOLO v2, YOLO v3 etc., which use a single network for efficiency.

In this paper, we use the faster R-CNN detector [62] with backbones such as (i) Inception V2 [73], (ii) Inception ResNet V2 [72], (iii) ResNet101 [30] and (iv) NASNet [97]. As the Inception V2, Inception ResNet V2 and NASNet are pre-trained on the COCO dataset [51], they detect from 91 object classes good at summarizing e.g., indoor environments and helping us associate the scene context with actions. The ResNet101 model is pre-trained on the AVA v2.1 dataset [27] with 80 different human actions, thus directly helping human-centric action recognition problems.

In addition to detection scores, we describe each bounding box with ImageNet [65] scores from pre-trained Inception ResNet V2 [72].

Saliency detectors. Image regions correlating with human visual attention are detected by saliency detectors in the form of saliency maps. Deep saliency models [86, 32] outperform conventional saliency detectors [96] but they require pixel-wise annotations. Recent models include MNL [95] (weakly-supervised model), RFCN [86] (a fully-supervised model) and a cheap non-CNN Robust Background Detector (RBD) [96] (see survey [2] for more details).

For the spatial saliency, we use MNL [95] trained on multiple noisy labels from weak/noisy unsupervised handcrafted saliency models. For temporal saliency, we use a CNN-LSTM ACLNet [94].

Deep learning AR. Early AR CNN models use frame-wise features and average pooling [36] discarding the temporal order. Thus, frame-wise CNN scores were fed to LSTMs [16] while the two-stream networks [70] compute representations per RGB frame and per 10 stacked optical flow frames. Finally, spatio-temporal 3D CNN filters [35, 75, 19, 78] model spatio-temporal patterns.

As two-stream networks [70] discard the temporal order, rank pooling [21, 22, 9, 82] and higherorder pooling [8, 38, 42, 17, 40] are popular. A recent I3D model [5] 'inflates' 2D CNN filters pre-trained on ImageNet to spatio-temporal 3D filters, and implements temporal pooling. PAN [93] proposes a motion cue called Persistence of Appearance that enables the network to distill the motion information directly from adjacent RGB frames. Approach [53] uses bootstrapping with long-range temporal context attention while approach [47] proposes a graph attention model to explore the semantics. Slow-I-Fast-P (SIFP) [50] for compressed AR contains the slow and fast pathways I and $\mathrm{P}$, resp., receiving a sparse sampling I-frame clip and a dense sampling pseudo optical flow clip.

AssembleNet [67] automatically finds a neural architecture with a good connectivity to capture spatio-temporal interactions for AR through NAS. AssembleNet++ [66] further learns the interactions between raw appearance and/or motion features and spatial object information through learning dynamic attention weights and search through the inter-block attention connectivity.

We use DEEP-HAL [85] which employs a 1D convolution for temporal pooling (I3D net.). We also investigate the use of AssembleNet and AssembleNet++ as backbones to show that our proposed object and saliency descriptors are independent of the backbone. We focus on the design/ability of ODF/SDF to supervise DEEP-HAL.

Power Normalization. For BoW/FV and CNN-based streams, the so-called burstiness defined as 'the property that a given visual element appears more times in an image than a statistically independent model would predict' [34] has to be tackled. Thus, we employ Power Normalization [43, 41, 42, 45, 44] which suppresses the burstiness via the so-called MaxExp pooling [43] given in Section 3.

\section{Background}

Below, we present Power Normalization [43, 42], count sketches [87], and the RBF feature maps which we use in our pipeline with the goal of the burstiness and dimensionality reduction, and Cartesian coordinate/frame positional encoding. 
Notations. We use boldface uppercase letters to express matrices e.g., $\boldsymbol{M}, \boldsymbol{P}$, regular uppercase letters with a subscript to express matrix elements $e . g ., P_{i j}$ is the $(i, j)^{\text {th }}$ element of $\boldsymbol{P}$, boldface lowercase letters to express vectors, e.g. $\mathbf{x}, \phi, \psi$, and regular lowercase letters to denote scalars. Vectors can be numbered e.g., $\mathrm{x}_{n}$ while regular lowercase letters with a subscript express an element of vector $e . g ., \mathbf{x}_{i}$ is the $i^{\text {th }}$ element of $\mathbf{x}$. Operators ';' and ',' concatenate vectors along the first and second mode, respectively, $\odot_{i \in \mathcal{I}_{K}} \mathbf{v}_{i}=\left[\mathbf{v}_{1} ; \ldots ; \mathbf{v}_{K}\right]$ and $\odot_{i \in \mathcal{I}_{K}}^{2} \mathbf{v}_{i}=\left[\mathbf{v}_{1}, \ldots, \mathbf{v}_{K}\right]$ concatenate a group of vectors in the first and second mode, respectively, $\oplus$ denotes the aggregation (sum) while $\mathcal{I}_{d}$ denotes an index set of integers $\{1, \ldots, d\}$.

\subsection{Power Normalization}

Proposition 1. Sigmoid (SigmE), a Max-pooling approximation [45], is an extension of the MaxExp operator defined as $g(\boldsymbol{\psi}, \eta)=1-(1-\boldsymbol{\psi})^{\eta}$ for $\eta>1$ to the operator with a smooth derivative, $a$ response defined for real-valued $\boldsymbol{\psi}$ (rather than $\psi \geq 0$ ), a parameter $\eta^{\prime}$ and a small constant $\epsilon^{\prime}$ :

$$
g\left(\boldsymbol{\psi}, \eta^{\prime}\right)=\frac{2}{1+e^{-\eta^{\prime} \boldsymbol{\psi} /\left(\|\boldsymbol{\psi}\|_{2}+\epsilon^{\prime}\right)}}-1 .
$$

Proof. See papers [43, 45] for extensive considerations.

As papers [43, 85] show that various pooling operators perform similarly, we equip our hallucination streams with SigmE followed by count sketching described below.

\subsection{Count Sketches}

Sketching vectors by the count sketch $[11,87]$ is used for their dimensionality reduction which we use in this paper.

Proposition 2. Let $d$ and $d^{\prime}$ denote the dimensionality of the input and sketched output vectors, respectively. Let vector $\boldsymbol{h} \in \mathcal{I}_{d^{\prime}}^{d}$ contain d uniformly drawn integer numbers from $\left\{1, \ldots, d^{\prime}\right\}$ and vector $s \in\{-1,1\}^{d}$ contain d uniformly drawn values from $\{-1,1\}$. Then, the sketch projection matrix $\boldsymbol{P} \in\{-1,0,1\}^{d^{\prime} \times d}$ becomes:

$$
P_{i j}= \begin{cases}s_{i} & \text { if } h_{i}=j \\ 0 & \text { otherwise }\end{cases}
$$

and the sketch projection $p: \mathbb{R}^{d} \rightarrow \mathbb{R}^{d^{\prime}}$ is a linear operation given as $p(\boldsymbol{\psi})=\boldsymbol{P} \boldsymbol{\psi}($ or $p(\boldsymbol{\psi} ; \boldsymbol{P})=\boldsymbol{P} \boldsymbol{\psi}$ to highlight $\boldsymbol{P}$ ).

Proof. It directly follows from the definition of the count sketch e.g., see Definition 1 [87].

Remark 1. Count sketches are unbiased estimators:

$\mathbb{E}_{\boldsymbol{h}, \boldsymbol{s}}\left(p(\boldsymbol{\psi}, \boldsymbol{P}(\boldsymbol{h}, \boldsymbol{s})), p\left(\boldsymbol{\psi}^{\prime}, \boldsymbol{P}(\boldsymbol{h}, \boldsymbol{s})\right)\right)=\left\langle\boldsymbol{\psi}, \boldsymbol{\psi}^{\prime}\right\rangle$. As variance

$\mathbb{V}_{\boldsymbol{h}, \boldsymbol{s}}\left(p(\boldsymbol{\psi}), p\left(\boldsymbol{\psi}^{\prime}\right)\right) \leq \frac{1}{d^{\prime}}\left(\left\langle\boldsymbol{\psi}, \boldsymbol{\psi}^{\prime}\right\rangle^{2}+\|\boldsymbol{\psi}\|_{2}^{2}\left\|\boldsymbol{\psi}^{\prime}\right\|_{2}^{2}\right)$, the larger sketches are less noisy. Thus, for every modality, we use a separate sketch matrix $\boldsymbol{P}$.

Proof. For the first and second property, see Appendix A of paper [87] and Lemma 3 [59].

\subsection{Positional Embedding}

Let $G_{\sigma}\left(\mathbf{x}-\mathbf{x}^{\prime}\right)=\exp \left(-\left\|\mathbf{x}-\mathbf{x}^{\prime}\right\|_{2}^{2} / 2 \sigma^{2}\right)$ denote a standard Gaussian RBF kernel centered at $\mathbf{x}^{\prime}$ and having a bandwidth $\sigma$. Kernel linearization refers to rewriting this $G_{\sigma}$ as an inner-product of two infinite-dimensional feature maps. To obtain these maps, we use a fast approximation method based on probability product kernels [33]. Specifically, we employ the inner product of $d^{\prime \prime}$-dimensional isotropic Gaussians given $\mathbf{x}, \mathbf{x}^{\prime} \in \mathbb{R}^{d^{\prime \prime}}$. Thus, we have:

$$
G_{\sigma}\left(\mathbf{x}-\mathbf{x}^{\prime}\right)=\left(\frac{2}{\pi \sigma^{2}}\right)^{\frac{d^{\prime \prime}}{2}} \int_{\boldsymbol{\zeta} \in \mathbb{R}^{d^{\prime \prime}}} G_{\sigma / \sqrt{2}}(\mathbf{x}-\boldsymbol{\zeta}) G_{\sigma / \sqrt{2}}\left(\mathbf{x}^{\prime}-\boldsymbol{\zeta}\right) \mathrm{d} \boldsymbol{\zeta} .
$$

Eq. (3) is then approximated by replacing the integral with the sum over $Z$ pivots $\boldsymbol{\zeta}_{1}, \ldots, \boldsymbol{\zeta}_{Z}$, thus yielding a feature map $\phi$ as: 


$$
\begin{aligned}
& \phi\left(\mathbf{x} ;\left\{\boldsymbol{\zeta}_{i}\right\}_{i \in \mathcal{I}_{Z}}\right)=\left[G_{\sigma / \sqrt{2}}\left(\mathbf{x}-\boldsymbol{\zeta}_{1}\right), \ldots, G_{\sigma / \sqrt{2}}\left(\mathbf{x}-\boldsymbol{\zeta}_{Z}\right)\right]^{T}, \\
& \quad \text { and } G_{\sigma}\left(\mathbf{x}-\mathbf{x}^{\prime}\right) \approx\left\langle\sqrt{c} \boldsymbol{\phi}(\mathbf{x}), \sqrt{c} \phi\left(\mathbf{x}^{\prime}\right)\right\rangle,
\end{aligned}
$$

where $c$ is a const. Eq. (5) is the linearization of the RBF kernel. Eq. (4) is the feature map. $\left\{\boldsymbol{\zeta}_{i}\right\}_{i \in \mathcal{I}_{Z}}$ are pivots. As we use $1 \mathrm{dim}$. signals, we simply cover interval $[0 ; 1]$ with $Z$ equally spaced pivots. For clarity, we drop $\left\{\boldsymbol{\zeta}_{i}\right\}_{i \in \mathcal{I}_{Z}}$ and write $\phi(\mathbf{x})$, etc.

\section{Approach}

Our pipeline is illustrated in Figure 2. It consists of (i) streams already present in DEEP-HAL [85] such as the FV/BoW streams (black), the High Abstraction Features (HAF) stream and the Optical Flow Features (OFF) which are fed into (ii) the Prediction Network abbreviated as PredNet. In this paper we focus on two non-trivial streams, that is the Object Detection Features and Saliency Detection Features (dashed blue) (ODF and SDF for short).

BoW/FV/OFF streams take the backbone intermediate representations generated from the RGB frames and learn to hallucinate BoW/FV and the optical flow (I3D only) representations via the MSE loss between the ground-truth BoW/FV/OFF and the outputs of BoW/FV/OFF streams. For AssembleNet/AssembleNet++, RGB and optical flow are combined by the backbone, thus we remove the OFF stream. The same MSE loss is applied to the ODF and SDF streams. However, the design of compact ground-truth ODF and SDF descriptors is one of our main contributions.

The HAF stream processes the backbone representations prior to combining them with the hallucinated streams. PredNet fuses the combined BoW/FV/OFF/HAF and our new ODF and SDF to learn actions on videos. Below, we start by describing how we obtain our ODF and SDF descriptors before we describe modules of DEEP-HAL [85] and our modifications. One change is that we learn weights for the weighted mean pooling (i.e., $\left.\sum_{i} w_{i}^{\prime} \psi / \sum_{i} w_{i}^{\prime}\right)$ of each stream to avoid concatenation of streams (prevent overparametrization).

\subsection{Statistical Motivation}

Before we outline our ODF and SDF descriptors, we motivate the use of higher-order statistics. To compare videos, we want to capture a distribution of local features/descriptors $e . g$., detection scores. The characteristic function $\varphi_{\Upsilon}(\boldsymbol{\omega})=\mathbb{E}_{\boldsymbol{v} \sim \Upsilon}\left(\exp \left(i \boldsymbol{\omega}^{T} \boldsymbol{v}\right)\right)$ describes the probability density $f_{\Upsilon}(\boldsymbol{v})$ of some video features (local features $\boldsymbol{v} \sim \Upsilon$ ). We obtain the Taylor expansion of the characteristic function:

$$
\begin{array}{r}
\mathbb{E}_{\boldsymbol{v} \sim r}\left(\sum_{r=0}^{\infty} \frac{i^{j}}{r !}\langle\boldsymbol{v}, \boldsymbol{\omega}\rangle^{r}\right) \approx \frac{1}{N} \sum_{n=0}^{N} \sum_{r=0}^{\infty} \frac{i^{r}}{r !}\left\langle\uparrow \otimes_{r} \boldsymbol{v}_{n}, \uparrow \otimes_{r} \boldsymbol{\omega}\right\rangle= \\
\sum_{r=0}^{\infty} \frac{i^{r}}{r !}\left\langle\frac{1}{N} \sum_{n=0}^{N} \uparrow \otimes_{r} \boldsymbol{v}_{n}, \uparrow \otimes_{r} \boldsymbol{\omega}\right\rangle=\sum_{r=0}^{\infty}\left\langle\mathcal{X}^{(r)}, \frac{i^{r}}{r !} \uparrow \otimes_{r} \boldsymbol{\omega}\right\rangle,
\end{array}
$$

where $i$ is the imaginary number, and a tensor descriptor $\mathcal{X}^{(r)}=\frac{1}{N} \sum_{n=0}^{N} \uparrow \otimes_{r} \boldsymbol{v}_{n}$. In principle, with infinite data and infinite moments, one can fully capture $f_{\Upsilon}(\boldsymbol{v})$. In practice, first-, second- and thirdorder moments are typically sufficient, however, second- and third-order tensors grow quadratically and cubically w.r.t. the size of $\boldsymbol{v}$. Thus, in what follows, we represent second-order moments not by a covariance matrix but by the subspace corresponding to the top $n^{\prime}$ leading eigenvectors. We also make use of the corresponding eigenvalues of the signal. Finally, it suffices to notice that $\boldsymbol{\kappa}^{(r)}=\operatorname{diag}\left(\boldsymbol{\mathcal { X }}^{(r)}\right)$ corresponds to the notion of order $r$ cumulants used in calculations of skewness $(r=3)$ and kurtosis $(r=4)$ but it grows linearly w.r.t. the size of $\boldsymbol{v}$. Thus, in what follows, we use the $\ell_{2}$ norm normalized mean, leading eigenvectors, trace-normalized eigenvalues, skewness and kurtosis (rather than coskewness and cokurtosis) to obtain compact representation of ODF and SDF.

\subsection{Object Detection Features}

Each object bounding box is described by the feature vector:

$$
\boldsymbol{v}=\left[\boldsymbol{\delta}\left(y_{(\text {det })}\right) ; \mathbf{y}_{(\text {inet })} ; \phi(\varsigma) ; \odot_{i \in \mathcal{I}_{4}} \phi\left(v_{i}\right) ; \phi\left(\frac{t-1}{\tau-1}\right)\right] \in \mathbb{R}^{d},
$$


where $\delta=[0, \ldots, 1, \ldots, 0]^{T}$ is a vector with all zeros but a single 1 placed at the location $y$. As we have 91 object classes for detectors trained on the COCO dataset and 80 classes for a detector trained on the AVA v2.1 dataset, we simply assume $y_{(\text {det })} \in \mathcal{I}_{91+80}$, that is, the labels $0, \ldots, 91$ describe classes from COCO while classes $92, \ldots, 80+91$ describe classes from AVA v2.1. Moreover, $\mathbf{y}_{(\text {inet })} \in \mathbb{R}^{1001}$ is an $\ell_{1}$ norm normalized ImageNet classification score, $0 \leq \varsigma \leq 1$ is the detector confidence score, $v_{0}, \ldots, v_{4}$ are the top-left and bottom-right Cartesian coordinates of a bounding box normalized in range $[0 ; 1]$, and $(t-1) /(\tau-1)$ is the frame index normalized w.r.t. the video sequence length $\tau$. For feature maps $\phi(\cdot)$ defined in Eq. (4), we simply use $Z=7$ pivots and the $\sigma$ of RBF is set to 0.5 . Finally, for all detections per video from a given detector, we first compute the mean $\boldsymbol{\mu}\left(\left[\boldsymbol{v}_{1}, \ldots, \boldsymbol{v}_{N}\right]\right) \in \mathbb{R}^{d}$ (we write $\boldsymbol{\mu}$ ) where $N$ is the total number of detections. Then, we form a matrix $\Upsilon \in \mathbb{R}^{d \times N}$ :

$$
\boldsymbol{\Upsilon}=\frac{1}{J}\left[\frac{1}{K_{1}}\left[\bigodot_{i \in \mathcal{I}_{K_{1}}}^{2}\left(\boldsymbol{v}_{i 1}-\boldsymbol{\mu}\right)\right], \ldots, \frac{1}{K_{J}}\left[\bigcirc_{i \in \mathcal{I}_{K_{J}}}^{2}\left(\boldsymbol{v}_{i J}-\boldsymbol{\mu}\right)\right]\right],
$$

where $K_{j}$ denotes a number of detections per frame $j \in \mathcal{I}_{J}$, from which we extract higher-order statistical moments as described below. As $N$ is large and its size varies from video to video, hallucinating $\Upsilon$ directly is not feasible (nor it has invariance properties).

Firstly, we obtain $\boldsymbol{U} \boldsymbol{\lambda} \boldsymbol{V}=\operatorname{svd}(\boldsymbol{\Upsilon})$ rather than $\boldsymbol{U} \boldsymbol{\lambda}^{2} \boldsymbol{U}^{T}=\operatorname{eig}\left(\boldsymbol{\Upsilon} \boldsymbol{\Upsilon}^{T}\right)$ as $N \ll d$, where $\boldsymbol{U}=$ $\left[\mathbf{u}_{1}, \mathbf{u}_{2}, \ldots\right]$. Take $\mathcal{X}^{(r)}\left(\{\mathbf{v}-\boldsymbol{\mu}\}_{n=0}^{N}\right)$ (which we abbreviate to $\boldsymbol{\mathcal { X }}^{(r)}$ ) and $\boldsymbol{\kappa}^{(r)}=\operatorname{diag}\left(\boldsymbol{\mathcal { X }}^{(r)}\right)$ defined in Section 4.1. We form our multi-moment descriptor $\psi_{(\text {det })} \in \mathbb{R}^{d\left(4+n^{\prime}\right)}, n^{\prime} \geq 1$ :

$$
\boldsymbol{\psi}_{(\text {det })}=\left[\frac{\boldsymbol{\mu}}{\|\boldsymbol{\mu}\|_{2}} ; \odot_{i \in \mathcal{I}_{n^{\prime}}}^{2} \mathbf{u}_{i}\left(\boldsymbol{\mathcal { X }}^{(2)}\right) ; \frac{\boldsymbol{\kappa}^{(3)}}{\left(\boldsymbol{\kappa}^{(2)}\right)^{3 / 2}} ; \frac{\boldsymbol{\kappa}^{(4)}}{\left(\boldsymbol{\kappa}^{(2)}\right)^{2}} ; \frac{\operatorname{diag}\left(\boldsymbol{\lambda}^{2}\right)}{\sum_{i} \lambda_{i i}^{2}}\right],
$$

The composition of Eq. (9) is described in Section 4.1. It is easy to verify that $\frac{\kappa^{(3)}}{\left(\kappa^{(2)}\right)^{3 / 2}}$ and $\frac{\kappa^{(4)}}{\left(\kappa^{(2)}\right)^{2}}$ are the empirical versions of skewness and kurtosis given by $\frac{\mathbb{E}_{\boldsymbol{v} \sim \Upsilon}\left((\boldsymbol{v}-\boldsymbol{\mu})^{3}\right)}{\mathbb{E}_{\boldsymbol{v} \sim r}^{3 / 2}\left((\boldsymbol{v}-\boldsymbol{\mu})^{2}\right)}$ and $\frac{\mathbb{E}_{\boldsymbol{v} \sim \Upsilon\left((\boldsymbol{v}-\boldsymbol{\mu})^{4}\right)}}{\mathbb{E}_{\boldsymbol{v} \sim \Upsilon}^{2}\left((\boldsymbol{v}-\boldsymbol{\mu})^{2}\right)}$.

\subsection{Saliency Detection Features}

We extract directional gradients from saliency frames by discretised gradient operators $[-1,0,1]$ and $[-1,0,1]^{T}$ and obtain gradient amplitude snd orientation maps $\boldsymbol{\Lambda}$ and $\boldsymbol{\theta}$ per frame encoded by:

$$
\boldsymbol{v}_{(s a l)}^{\prime}=\sum_{i \in \mathcal{I}_{W}, j \in \mathcal{I}_{H}} \Lambda_{i j} \phi\left(\theta_{i j} /(2 \pi)\right) \otimes \phi\left(\frac{i-1}{W-1}\right) \otimes \phi\left(\frac{j-1}{H-1}\right),
$$

where $\otimes$ is the Kronecker product and $\phi(\theta)$ follows Eq. (4) with the exception that the assignment to Gaussians is realized in the modulo ring to respect the periodical nature of $\theta$. We encode $\phi(\theta)$ with 12 pivots which encode the orientation of gradients. The remaining maps $\phi(\cdot)$ are encoded with 5 pivots each, which correspond to spatial binning. Note that $\boldsymbol{v}_{(s a l)}^{\prime}$ (we write $\left.\boldsymbol{v}^{\prime}\right)$ is similar to a single CKN layer [54] but is simpler: for one dimensional variables we sample pivots (c.f. learn) for maps $\phi(\cdot)$. Each saliency frame is described as a feature vector $\boldsymbol{v}^{\dagger}=\left[\boldsymbol{v}^{\prime} /\left\|\boldsymbol{v}^{\prime}\right\|_{2} ; \mathbf{I}_{:} /\left\|\mathbf{I}_{:}\right\|_{1}\right] \in \mathbb{R}^{d^{\dagger}}$, where $\mathbf{I}_{\text {: }}$ is a vectorized low-resolution saliency map. Thus, $\boldsymbol{v}^{\dagger}$ captures the directional gradient statistics and the intensity-based gist of saliency maps. Subsequently, we compute the mean $\boldsymbol{\mu}\left(\left[\boldsymbol{v}_{1}^{\dagger}, \ldots, \boldsymbol{v}_{J}^{\dagger}\right]\right) \in \mathbb{R}^{d^{\dagger}}$ (we simply write $\boldsymbol{\mu}$ ) where $J$ is the total number of frames per video. Then, we obtain $\boldsymbol{\Upsilon}^{\dagger}=$ $\left[\boldsymbol{v}_{1}^{\dagger}, \ldots, \boldsymbol{v}_{J}^{\dagger}\right] / J \in \mathbb{R}^{d^{\dagger} \times J}$ which is compactly described by the multi-moment Eq. (9) resulting in $\boldsymbol{\psi}_{(s a l)} \in \mathbb{R}^{d\left(4+n^{\dagger}\right)}$.

\subsection{Hallucinating Streams/High Abstr. Features}

Each hallucinating stream takes as input the backbone intermediate representation $\mathcal{X}_{(\mathrm{rgb})}$ of size $1024 \times 7$ obtained by removing the classifier and the last 1D conv. layer of I3D pre-trained on Kinetics-400. For AssembleNet/AssembleNet++, instead of the classification layer (FC layer), we use a 2048 dimensional output from 3D AveragePooling layer. For the BoW/FV/OFF and HAL streams, we follow the steps described in the DEEP-HAL approach [85]. For all streams, we use 


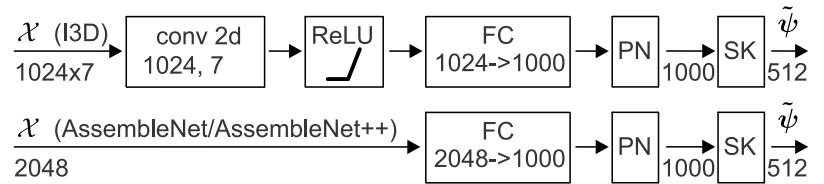

(a)

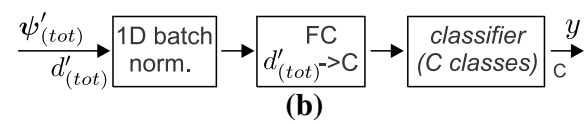

Figure 3: Stream details. Figure $3 \mathrm{a}$ a shows the stream architecture used by us for the FV, BoW, OFF, HAF, DET1, ...,DET4, SAL1 and SAL2 streams. Figure 3b shows our PredNet. Operation and their parameters are in each block e.g., conv2d and its number of filters/size, Power Normalization $(P N)$ and Sketching $(S K)$. We indicate the size of input and/or output under arrows.

a Fully Connected (FC) unit shown in Figure 3a. Each stream uses Power Normalization (PN) realized via SigmE and Sketching (SK) from 1000 to $512 \operatorname{dim}$ via $\tilde{\psi}_{(\cdot)}^{\prime}=\tilde{\boldsymbol{P}}_{(\cdot)} \tilde{\boldsymbol{\psi}}_{(\cdot)}$. Outputs $\tilde{\boldsymbol{\psi}}^{\prime}$ can be now aligned with ground-truth $\psi_{(\cdot)}^{\prime}$ described below. The same steps are applied to High Abstraction Features (HAF), combined with other streams, and also fed into PredNet (see Fig. 2). While hallucinating streams co-supervise the backbone via external ground-truth tasks, HAF simply passes the backbone features into PredNet.

Ground-truth BoW/FV/OFF. We follow the DEEP-HAL setup [85] and apply PCA to a concatenation of IDT trajectories (30 dim.), HOG (96 dim.), HOF (108 dim.), MBHx (96 dim.) and MBHy (96 dim.). The resulting $213 \mathrm{dim}$. local descriptors are encoded by FV and BoW with a $256 \mathrm{dim}$. and a $1000 \mathrm{dim}$. GMM and k-means dictionaries. For the OFF stream (not used with AssembleNet or AssembleNet++), we pre-computed I3D with LDOF $\mathcal{X}_{(\text {opt. }}$ (Fig. 2). All ground-truth representations were Power Normalized by SigmE/sketched to $512 \mathrm{dim}$. each via $\boldsymbol{\psi}_{(\cdot)}^{\prime}=\boldsymbol{P}_{(\cdot)} \psi_{(\cdot)}$ and fed into the MSE loss. No ground-truth testing data is used in training/testing.

Ground-truth DET1, ...,DET4/SAL1/SAL2. The ODF ground-truth training representations are of size $1214 \times N$, where $N$ is the total number of bounding boxes per video (50-10000). The feature dim. 1214 is composed of $80+91 \mathrm{dim}$. one-hot detection classes, $6 \times 7$ are the $\phi(\cdot)$-embedded confidence, bounding box coordinates and the frame number, 1001 is the ImageNet score. We also consider a variant without the RBF embedding: $\phi(\mathbf{x})=\mathbf{x}(1178 \times N$ size $)$. The SDF ground-truth training repr. are of size $556 \times J$, where $J$ is the number of frames per video. $300 \mathrm{dim}$. $(12 \times 5 \times 5)$ concern spatio-angular gradient distributions and $256 \mathrm{dim} .(16 \times 16)$ concern the luminance of saliency maps. Each ODF/SDF is encoded per video with the multi-moment descriptor in Eq. (9) yielding $1178 \times\left(4+n^{\prime}\right)$ and $556 \times\left(4+n^{\dagger}\right)$ compact representations (we vary $n^{\prime}$ and $n^{\dagger}$ between 1 and 5). ODF and SDF are Power Normalized by SigmE/sketched to $512 \mathrm{dim}$. each via $\boldsymbol{\psi}_{(\cdot)}^{\prime}=\boldsymbol{P}_{(\cdot)} \boldsymbol{\psi}_{(\cdot)}$ and fed into the MSE loss. No ground-truth testing representations were used for training/testing.

\subsection{Objective Function}

During training, we combine MSE loss functions which co-supervise hallucination streams with the classifier:

$$
\begin{aligned}
\ell^{*}(\boldsymbol{\mathcal { X }}, \mathbf{y} ; \overline{\boldsymbol{\Theta}})=\frac{\alpha}{|\mathcal{H}|} \sum_{i \in \mathcal{H}}\left\|\tilde{\boldsymbol{\psi}}_{i}-\boldsymbol{\psi}_{i}^{\prime}\right\|_{2}^{2}+\ell\left(f\left(\boldsymbol{\psi}_{(t o t)}^{\prime} ; \boldsymbol{\Theta}_{(p r)}\right), \mathbf{y} ; \boldsymbol{\Theta}_{(\ell)}\right), \\
\text { where: } \forall i \in \mathcal{H}, \tilde{\boldsymbol{\psi}}_{i}^{\prime}=\tilde{\boldsymbol{P}}_{i} g\left(\hbar\left(\mathcal{X}, \boldsymbol{\Theta}_{i}\right), \eta\right), \boldsymbol{\psi}_{i}^{\prime}=\boldsymbol{P}_{i} \boldsymbol{\psi}_{i}, \\
\boldsymbol{\psi}_{(h a f)}^{\prime}=\boldsymbol{P}_{(h a f)} g\left(\hbar\left(\boldsymbol{\mathcal { X }}, \boldsymbol{\Theta}_{(h a f)}\right), \eta\right) \\
\boldsymbol{\psi}_{(t o t)}^{\prime}=\frac{1}{\left|\mathcal{H}^{*}\right|+1}\left(w_{(h a f)} \boldsymbol{\psi}_{(h a f)}^{\prime}+\sum_{i \in \mathcal{H}^{*}} w_{i} \tilde{\boldsymbol{\psi}}_{i}^{\prime}\right) \\
\tilde{\boldsymbol{\psi}}_{(\text {det })}^{\prime}=\frac{1}{|\mathcal{D}|} \sum_{i \in \mathcal{D}} w_{i} \tilde{\boldsymbol{\psi}}_{i}^{\prime}, \tilde{\boldsymbol{\psi}}_{(s a l)}^{\prime}=\frac{1}{|\mathcal{S}|} \sum_{i \in \mathcal{S}} w_{i} \tilde{\boldsymbol{\psi}}_{i}^{\prime}
\end{aligned}
$$

The above equation is a trade-off between the MSE loss functions $\left\{\left\|\tilde{\boldsymbol{\psi}}_{i}^{\prime}-\boldsymbol{\psi}_{i}^{\prime}\right\|_{2}^{2}, i \in \mathcal{H}\right\}$ and the classification loss $\ell\left(\cdot, \mathbf{y} ; \boldsymbol{\Theta}_{(\ell)}\right)$ with some label $\mathbf{y} \in \mathcal{Y}$ and parameters $\boldsymbol{\Theta}_{(\ell)} \equiv\{\boldsymbol{W}, \mathbf{b}\}$. The trade-off is controlled by $\alpha \geq 0$ while MSE is computed over hall. streams $i \in \mathcal{H}$, and $\mathcal{H} \equiv$ 
$\{(f v 1),(f v 2),($ bow $),($ of $f),(\operatorname{det} 1), \ldots,(\operatorname{det} 4),($ sal 1$),($ sal 2$)\}$ is our set of hallucination streams. Moreover, $g(\cdot, \eta)$ is a Power Norm. in Eq. (1), $f\left(\cdot ; \boldsymbol{\Theta}_{(p r)}\right)$ is the PredNet module with parameters $\boldsymbol{\Theta}_{(p r)}$ which we learn, $\left\{\hbar\left(\cdot, \boldsymbol{\Theta}_{i}\right), i \in \mathcal{H}\right\}$ are the hallucination streams while $\left\{\tilde{\boldsymbol{\psi}}_{i}, i \in \mathcal{H}\right\}$ are resulting hallucinated BoW/FV/OFF/ODF/SDF representations. We set $\alpha=1$. Moreover, $\hbar\left(\cdot, \boldsymbol{\Theta}_{(\text {haf })}\right)$ is the HAF stream with the sketched output $\boldsymbol{\psi}_{(\text {haf })}^{\prime}=\boldsymbol{P}_{(\text {haf })} \boldsymbol{\psi}_{(\text {haf })}$. For the hallucination streams, we learn parameters $\left\{\boldsymbol{\Theta}_{i}, i \in \mathcal{H}\right\}$ while for HAF, we learn $\boldsymbol{\Theta}_{(\text {haf })}$. The full set of parameters we learn is defined as $\overline{\boldsymbol{\Theta}} \equiv\left(\left\{\boldsymbol{\Theta}_{i}, i \in \mathcal{H}\right\}, \boldsymbol{\Theta}_{(h a f)}, \boldsymbol{\Theta}_{(p r)}, \boldsymbol{\Theta}_{(\ell)}\right)$. Furthermore, $\left\{\tilde{\boldsymbol{P}}_{i}, i \in \mathcal{H}\right\}$ and $\left\{\boldsymbol{P}_{i}, i \in \mathcal{H}\right\}$ are the projection matrices for count sketching of streams $\left\{\tilde{\boldsymbol{\psi}}_{i}, i \in \mathcal{H}\right\}$ and the ground-truth feature vectors $\left\{\boldsymbol{\psi}_{i}, i \in \mathcal{H}\right\}$. Finally, for $\boldsymbol{\psi}_{(t o t)}^{\prime}$ is a weighted average of several streams fed into the PredNet module $f$. Moreover, $\mathcal{H}^{*} \equiv\{(f v 1),(f v 2),($ bow $),($ of $f),(\operatorname{det}),($ sal $)\}, \mathcal{D} \equiv\{(\operatorname{det} 1), \ldots,(\operatorname{det} 4)\}$ and $\mathcal{S} \equiv\{($ sal1 $),($ sal2 $)\}$. Section 3.2 details how to select matrices $\boldsymbol{P}$. Let $\mathcal{T}$ be set to either $\mathcal{H}^{*}$, $\mathcal{D}$ or $\mathcal{S}$, then our weights are:

$$
w_{i}=\frac{1}{|\mathcal{T}|} \frac{\max \left(w_{i}^{\prime \beta}, \rho\right)}{\sum_{j \in \mathcal{T}} \max \left(w_{j}^{\prime \beta}, \rho\right)} .
$$

Prior to CNN training, we train an SVM on each ground-truth stream separately (using a manageable training subset), and we set weights $w^{\prime}$ proportionally to the accuracies obtained on the validation set. For the HAF stream, we simply set $w_{(h a f)}^{\prime}=\frac{1}{\left|\mathcal{H}^{*}\right|+1}$ and $\rho=0.1$. For the first few epochs (i.e., 10 ), we set $\beta=0$ so that all streams receive equal weights. Subsequently, in each epoch, we run the Golden-section search to find the best $\beta \geq 0$. We start from initial boundary values $\beta \in\{0,50\}$, we train an SVM on a manageable subset of training data and evaluate $\beta$ on the validation set, and we update boundary values for the next epoch accordingly. Eq. (12) has a nice property: for $\beta=0$, we have $w_{i}=1 /|\mathcal{T}|$. For $\beta \rightarrow \infty$, we have $w_{i}=1$ if $w_{i}=\max \left(\left\{w_{i}\right\}_{i \in \mathcal{T}}\right)$, otherwise $w_{i}=0$. Thus, $\beta$ interpolates between equalizing all weights and the winner-takes-all solution.

\section{Experiments}

\subsection{Datasets and Evaluation Protocols}

HMDB-51 [46] has 6766 internet videos/ 51 classes; each video has 20-1000 frames. We report the mean accuracy across three splits.

YUP++ [20] has 20 scene classes of video textures, 60 videos per class. Splits contain scenes captured by the static or moving camera. We use standard splits (1/9 dataset for training) for evaluation.

MPII Cooking Activities [64] contains high-resolution videos of people cooking dishes. The 64 activities from 3748 clips include coarse actions e.g., opening refrigerator, and fine-grained actions e.g., peel, slice, cut apart. We use the mean Average Precision (mAP) over 7-fold cross validation. For human-centric protocol [7, 9], we use the faster RCNN [62] to crop video around human subjects.

Charades [69] consist of of 9848 videos of daily indoors activities, 66500 clip annotations and 157 classes.

EPIC-Kitchens [14] is a multi-class egocentric dataset with $28 \mathrm{~K}$ training videos associated with 352 noun and 125 verb classes. The dataset consists of 39,594 segments in 432 videos. We follow protocol [1]. We evaluate our model on validation, standard seen (S1: 8047 videos), and unseen (S2: 2929 videos) test sets.

\subsection{Evaluations}

Below, we show the effectiveness of our method. For smaller datasets, we use the I3D backbone. For large Charades and EPIC-Kitchens, we additionally investigate AssembleNet and AssembleNet++ backbones. Firstly, we evaluate various design components.

Ground-truth ODF+SVM. Firstly, we evaluate our ODF on SVM given the HMDB-51 dataset. We set $n^{\prime}=3$ for Eq. (9) and compare various detector backbones and pooling strategies. Table 1 shows that all detectors perform similarly with $(\operatorname{det} 3)$ being slightly better than other methods. Moreover, max-pooling on ODFs from all four detectors is marginally better than the average-pooling. However, only the weighted mean (all+wei) according to Eq. (12) outperforms (det3) which highlights 


\begin{tabular}{|c|c|c|c|}
\hline & spl & $\operatorname{sp2}$ & mean acc. \\
\hline$\overline{\operatorname{det} 1}$ & $42.00 \%$ & $39.74 \% 40.39 \%$ & $40.72 \%$ \\
\hline detl & 40.4 & $40.13 \% 39.67 \%$ & 40 \\
\hline $\operatorname{det} 3$ & 43.7 & $\% 41.97 \%$ & 43 \\
\hline $\operatorname{det} 4$ & 41.0 & $\% 40.39 \%$ & 40 \\
\hline$a \bar{l} l+a$ & $\overline{42.50}$ & $5 \% \overline{4} 1.0 \overline{1} \%$ & $-\overline{41.5} \overline{2} \%$ \\
\hline all $+n$ & 43.25 & $42.32 \% 42.09 \%$ & $42.55 \%$ \\
\hline$a l l+w e i$ & 45.8 & $44.52 \% 44.09 \%$ & $44.80 \%$ \\
\hline$\overline{D E} \bar{P}-\bar{H}$ & $83.25 \%$ & $82.2 \overline{4} \% \overline{8} 2.84 \%$ & $-82.7 \overline{7} \%$ \\
\hline DEEP-HA & 091 & $1.86 \% 82.84 \%$ & $82.62 \%$ \\
\hline & & & \\
\hline
\end{tabular}

Table 1: Evaluations of ODF on HMDB-51. (top) We evaluate backbones such as (det 1$)$ Inception V2, (det2) Inception ResNet V2, (det3) ResNet101 and (det4) NASNet. (middle) The average-pooled, max-pooled and the weighted mean combination of all detectors are given by $($ all $+a v g),(a l l+m a x)$ and $($ all + wei $)$. (bottom) Pretrained DEEP-HAL combined with all four detectors by the average-pooling, max-pooling and the weighted mean.

\begin{tabular}{lccc}
\hline & avg & max & wei \\
\hline all & $55.12 \%$ & $42.34 \%$ & $\mathbf{6 0 . 5 2} \%$ \\
DEEP-HAL+all & $74.22 \%$ & $71.85 \%$ & $\mathbf{7 5 . 7 4} \%$
\end{tabular}

Table 2: Pooling on YUP++. Results for the average-pooled (avg), max-pooled ( $\max$ ) and the weighted mean (wei) of all detectors (all) vs. pre-trained DEEP-HAL combined with all detectors by the average-pooling, max-pooling and the weighted mean.

the need for the robust aggregation of ODFs. Similarly, when we combine pre-trained DEEP-HAL with all detectors, the weighted mean (DEEP-HAL+all+wei) performs best. Table 2 shows the similar trend on YUP++. We trained SVM only on videos for which at least one detection occurred, thus a $75.74 \%$ accuracy is much lower than the main results reported on the full pipeline. Figure 4 shows that $\beta \neq 1$ has a positive impact on reweighting.

Ground-truth SDF. The SDF on HMDB-51 and YUP++ yielded $24.35 \%$ and $32.68 \%$ accuracy. This is expected as SDFs do not capture a discriminative information per se but they locate salient spatial and temporal regions to focus the main network on them.

Multi-moment descr. Figure 5 shows that the concat. of the mean and three eigenvectors according to Eq. (9) yields good results but adding further vectors deteriorates the performance. Adding skewness and kurtosis ( $\varsigma$ and $\varphi$ ) further improves results, while adding eigenvalues has a limited impact.

HMDB-51. Table 3 shows several DEEP-HAL variants, which all hallucinate BoW/FV/OFF. DEEP-HAL with our reweighting mechanism. $(D E E P-H A L+W)$ outperforms the original DEEPHAL denoted as $(H A F / B o W / F V$ hal. $)$ [85] by $\sim 0.8 \%$. DEEP-HAL with our ODF and SDF descriptors $(D E E P-H A L+O D F)$ and $(D E E P-H A L+S D F)$ outperform $(H A F / B o W / F V h a l$. $)$ by $\sim 1.8 \%$ and $\sim 1.4 \%$, resp. This shows that both ODF and SDF are effective. Combining DEEP-HAL,

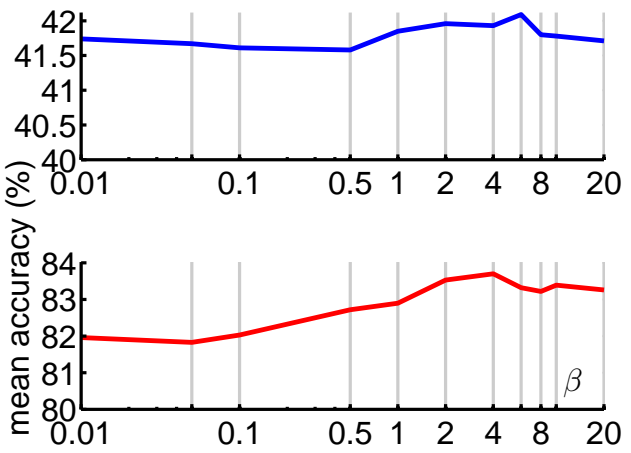

(a)

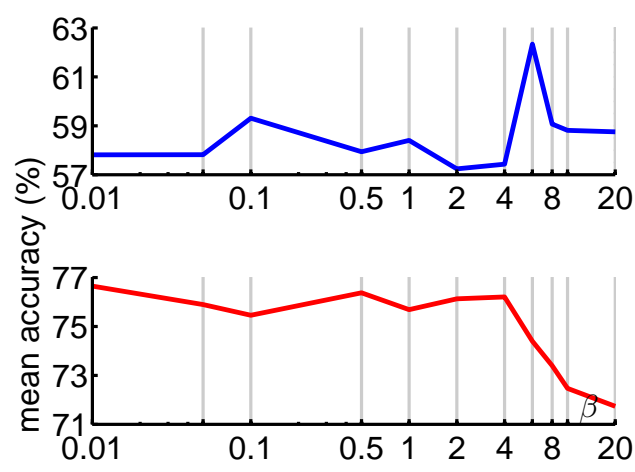

(b)

Figure 4: The impact of $\beta$ in the weighted mean on the classification results. Figure $4 \mathrm{a}$ shows results for HMDB-51 on (top) four detectors combined+SVM and (bottom) DEEP-HAL with four detectors combined+SVM. Figure $4 \mathrm{~b}$ shows results for YUP++. 


\begin{tabular}{lcccc}
\hline & $s p 1$ & $s p 2$ & $s p 3$ & mean acc. \\
\hline$D E E P-H A L+W$ & $83.94 \%$ & $82.50 \%$ & $83.34 \%$ & $83.26 \%$ \\
$D E E P-H A L+O D F$ & $85.03 \%$ & $83.59 \%$ & $84.25 \%$ & $84.29 \%$ \\
$D E E P-H A L+S D F$ & $84.64 \%$ & $83.20 \%$ & $83.82 \%$ & $83.88 \%$ \\
DEEP-HAL+ODF+SDF & $86.14 \%$ & $83.66 \%$ & $85.81 \%$ & $85.20 \%$ \\
DEEP-HAL+W+ODF+SDF & $87.78 \%$ & $86.27 \%$ & $87.06 \%$ & $87.04 \%$ \\
DEEP-HAL+W+G+ODF+SDF & $88.37 \%$ & $86.80 \%$ & $87.52 \%$ & $\mathbf{8 7 . 5 6} \%$ \\
\hline
\end{tabular}

ADL+I3D 81.5\% [82] Full-FT I3D 81.3\% [5]

EvaNet (Ensemble) 82.3\% [60] PA3D + I3D 82.1\% [91]

HAF/BoW/FV exact $82.50 \%$ [85] HAF/BoW/FV hal. $82.48 \%$ [85]

Table 3: Evaluations of (top) our methods and (bottom) comparisons to the state of the art on HMDB-51.

\begin{tabular}{lccccc}
\hline & static & dynamic & mixed & $\begin{array}{c}\text { mean } \\
\text { stat/dyn }\end{array}$ & $\begin{array}{c}\text { mean } \\
\text { all }\end{array}$ \\
\hline DEEP-HAL+ODF & $95.00 \%$ & $90.93 \%$ & $93.52 \%$ & $93.0 \%$ & $93.2 \%$ \\
$D E E P-H A L+S D F$ & $94.96 \%$ & $89.93 \%$ & $93.58 \%$ & $92.4 \%$ & $92.8 \%$ \\
$D E E P-H A L+S D F+O D F$ & $95.10 \%$ & $91.11 \%$ & $93.61 \%$ & $93.1 \%$ & $93.3 \%$ \\
$D E E P-H A L+W+S D F+O D F$ & $96.30 \%$ & $92.22 \%$ & $94.17 \%$ & $94.3 \%$ & $94.2 \%$ \\
$D E E P-H A L+W+G+S D F+O D F$ & $96.30 \%$ & $\mathbf{9 2 . 4 0} \%$ & $\mathbf{9 4 . 3 5 \%}$ & $\mathbf{9 4 . 4} \%$ & $\mathbf{9 4 . 4} \%$ \\
\hline T-ResNet [20] & $92.4 \%$ & $81.5 \%$ & $89.0 \%$ & $87.0 \%$ & $87.6 \%$ \\
ADL I3D [82] & $95.1 \%$ & $88.3 \%$ & - & $91.7 \%$ & - \\
HAF/BoW/FV hal. [85] & $94.8 \%$ & $89.6 \%$ & $93.3 \%$ & $92.2 \%$ & $92.6 \%$ \\
MSOE-two-stream [28] & $\mathbf{9 7 . 0} \%$ & $87.0 \%$ & $91.8 \%$ & $92.0 \%$ & $91.9 \%$ \\
\hline
\end{tabular}

Table 4: Evaluations of (top) our methods and (bottom) comparisons to the state of the art on YUP++.

ODF and SDF outperform DEEP-HAL by $\sim 2.7 \%$ demonstrating the complementary nature of ODF and SDF. Utilizing our weighting mechanism with DEEP-HAL, ODF and SDF denoted as $(D E E P-H A L+W+O D F+S D F)$ outperform $(H A F / B o W / F V$ hal. $)$ by $\sim 4.6 \%$. Finally, DEEP-HAL with weighting, and ODF and SDF with RBF feature maps from Eq. (4) outperform (HAF/BoW/FV hal.) by $\sim 5.1 \%$.

YUP++. Table 4 shows that ODF is better than SDF, that is $(D E E P-H A L+O D F)$ and $(D E E P$ $H A L+S D F)$ outperform $(H A F / B o W / F V$ hal. $)$ by $\sim 0.6 \%$ and $\sim 0.2 \%$, resp. This is expected as YUP++ contains dynamic scenes without objects/specific saliency regions correlating with class concepts. However, a combination of detectors/saliency $(D E E P-H A L+S D F)$ plus weighting $(D E E P-H A L+W+O D F+S D F)$ plus the RBF maps $(D E E P-H A L+W+G+O D F+S D F)$ outperform (HAF/BoW/FV hal. $)$ by $\sim 0.7 \%, \sim 1.6 \%$ and $\sim 1.8 \%$ accuracy, resp.

MPII. Table 5 shows a $\sim 3.0 \%$ mAP gain over $(H A F / B o W / F V$ hal. $)$ due to detectors capturing the human interaction with objects.

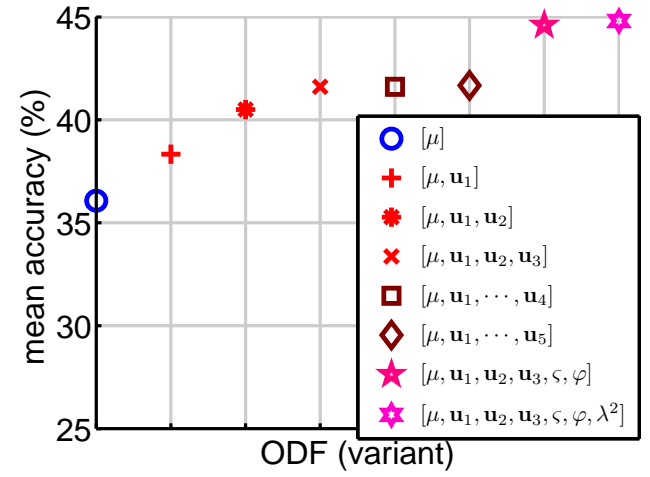

(a)

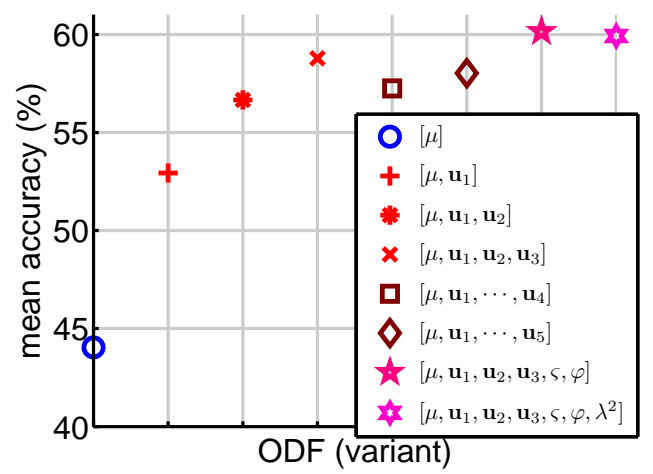

(b)

Figure 5: ODF eval. on SVM on four detectors (the weighted mean). Fig. 5a and 5b show results on HMDB-51 and YUP++. $\boldsymbol{\mu}, \mathbf{u}_{1}, \ldots, \mathbf{u}_{i}, \boldsymbol{\varsigma}, \boldsymbol{\varphi}$, and $\boldsymbol{\lambda}^{2}$ correspond to the entries in Eq. (9). 


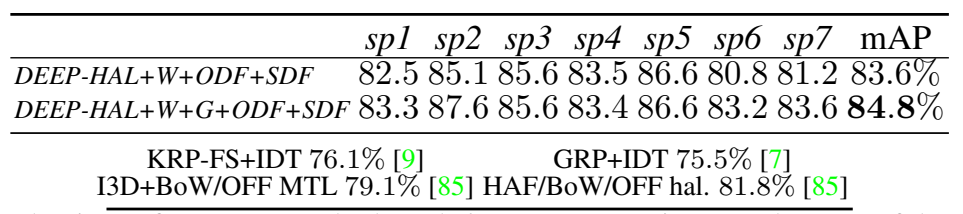

Table 5: Evaluations of (top) our methods and (bottom) comparisons to the state of the art on MPII.

Charades. Table 6 (top) presents relative gains of our hallucination pipeline (DEEP-HAL) with weighted mean pooling $(W)$ and the RBF maps $(G)$ denoted as $(D E E P-H A L+W+G)$. We evaluate Object Detection Features $(O D F)$ and Saliency Detection Features $(S D F)$ with 512 dim. sketching (SK512) and note that (DEEP-HAL+W+G+ODF (SK512)) outperforms (DEEP-HAL+W+G+SDF (SK512)), and both methods outperform the baseline (HAF/BoW/FV hal.) [85].

Table 6 (bottom) shows that combining ODF and SDF into (DEEP-HAL+W+G+SDF+ODF (SK512)) yields $49.06 \% \mathrm{mAP}$ which constitutes on a $\sim \mathbf{6} \%$ gain over the baseline $(H A F / B o W / F V$ hal.) [85]. This demonstrates that ODF and SDF are highly complementary. Applying a larger sketch $(D E E P-H A L+W+G+O D F+S D F(S K 1024))$ yields $50.14 \% \mathrm{mAP}$ which matches the use $(D E E P-H A L+W+G+O D F+S D F($ exact $))$ that denotes a late fusion by concatenation of ODF and SDF with the stream resulting from DEEP-HAL fed into PredNet. Note that (exact) indicates that ODF and SDF are not hallucinated at the test time but they are computed. the results matching between $(D E E P-H A L+W+G+O D F+S D F(S K 1024))$ and $(D E E P-H A L+W+G+O D F+S D F($ exact $))$ show that we can hallucinate ODF and SDF at the test time while regaining the full performance. We save computational time and hallucinate the detection and saliency features which boost results on Charades by $\sim \mathbf{6} \%$ over the baseline.

Table 7 shows that our idea applied to AssembleNet and AssembleNet++ yields state of the art e.g., we outperform these two networks by $\mathbf{4 . 5} \%$ and $\mathbf{5 . 6} \% \mathrm{mAP}$, respectively. We note that our detectors do not need to be computed at all at the test time.

In contrast, the best currently reported papers such as SlowFast networks [18] and AssembleNet [67] achieve $45.2 \%$ and 51.6\% on Charades. As SlowFast networks and AssembleNet backbones can be used in place of I3D in our experimental setup, our approach is 'orthogonal' to these latest developments which focus on heavy mining for combinations of neural blocks/dataflow between them to obtain an 'optimal' pipeline. We achieve similar results with a simple approach based on self-supervised learning. Our pipeline is lightweight by comparison (no need for computations of the optical flow, or detections or segmentation masks at test time).

ImageNet (global score) vs. object detectors. Various scores from the object and saliency detectors which we use cannot be plugged directly into the DEEP-HAL due to the varying number of objects detected and the varying number of frames, thus we propose and use ODF and SDF descriptors. We also note that using a simplified variant of ODF which stacks up ImageNet scores per frame into a matrix (no detectors) to which we apply our multi-moment descriptor yielded $\sim 4 \%$ worse results on Charades than our DEEP-HAL+ODF (detectors-based approach) which yields $48.0 \%$ mAP. This is expected as ImageNet is trained in a multi-class setting (one object per image) while detectors let us model robustly distributions of object classes and locations per frame.

EPIC-Kitchens.Table 8 shows the experimental results. I3D and AssembleNet/AssembleNet++ learn human-like semantic features due to ODF/SDF, and there is no evidence a backbone can discover these without a guidance. By comparing MPII (3748 clips) with large EPIC-Kitchens (39594 clips) (both about cooking), SDF+ODF boost MPII from 81.8 to $84.8 \%$, and SDF+ODF boost EPIC-Kitchens from $32.51 \%$ (DEEP-HAL) to $35.88 \%$ (on seen classes protocol), and from $22.33 \%$ (DEEP-HAL) to $27.32 \%$ (on unseen classes protocol). The boost is $\mathbf{3} \%$ on both MPII and EPIC-Kitchens (nearly $10 \times$ more clips than MPII).

\section{Conclusions}

We have introduced two simple yet effective object and saliency descriptors, which perform selfsupervision of an AR hallucination-based network. We have shown that modeling high-order statistical moments can result in small representations that can self-supervise our AR pipeline. The findings are in line with recent multi-task learning papers which argue that related tasks can co- 


\begin{tabular}{|c|c|}
\hline $\begin{array}{l}\text { HAF/BoW/FV } \\
\text { hal. [85] }\end{array}$ & $\begin{array}{cc}\text { DEEP-HAL+ } & \text { DEEP-HAL+ } \\
W+G+O D F(S K 512) & W+G+S D F(S K 512)\end{array}$ \\
\hline 43.1 & 47.22 \\
\hline
\end{tabular}

Table 6: Evaluations of our methods on Charades (I3D backbone).

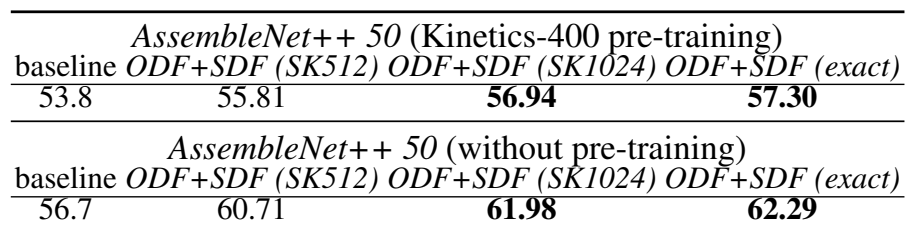

Table 7: Evaluations of our methods on the Charades dataset (AssembleNet and AssembleNet++ backbones). Note that we do not use segmentation masks for AssembleNet and AssembleNet++, thus baseline results reported by us are slightly lower compared to authors' results of $55.0 \%$ and $59.8 \%$ mAP, respectively.

\begin{tabular}{|c|c|c|c|c|c|c|}
\hline & \multicolumn{2}{|c|}{ Verbs } & \multicolumn{2}{|c|}{ Nouns } & \multicolumn{2}{|c|}{ Actions } \\
\hline & top-1 & top-5 & top-1 & top-5 & top-1 & top-5 \\
\hline & \multicolumn{6}{|c|}{ Validation } \\
\hline LFB Max [90] & 52.6 & 81.2 & 31.8 & 56.8 & 22.8 & 41.1 \\
\hline WeakLargeScale [24] & 58.4 & 84.1 & 36.9 & 60.3 & 26.1 & 42.7 \\
\hline$\overline{\mathrm{DE}} \overline{\mathrm{EP}}-\overline{\mathrm{H}} \overline{\mathrm{AL}}+\overline{\mathrm{O}} \overline{\mathrm{D}} \overline{\mathrm{F}}+\overline{\mathrm{S}} \overline{\mathrm{D}} \overline{\mathrm{F}}(\overline{\mathrm{SK}} \overline{\mathrm{O}} \overline{2} \overline{4})$ & $5 \overline{5} . \overline{4}$ & $-\overline{82 . \overline{9}}$ & $33.3^{-}$ & 55.1 & 21.5 & $\overline{3} \overline{9} . \overline{7}$ \\
\hline AssembleNet++ ODF+SDF(SK512) & 57.2 & 84.6 & 34.8 & 56.4 & 23.2 & 41.3 \\
\hline AssembleNet++ ODF+SDF(SK1024) & 58.7 & 85.6 & 36.0 & 57.3 & 24.7 & 43.0 \\
\hline \multirow[t]{2}{*}{$\overline{\mathrm{Assemble}} \overline{\mathrm{N}} \overline{\mathrm{et}}++\overline{\mathrm{ODF}} \overline{\mathrm{SD}} \overline{\mathrm{S}} \overline{(\mathrm{exact})} \overline{-}$} & $-6 \overline{0} . \overline{0}$ & $86 . \overline{7}$ & $37.1^{-}$ & $59.2^{-}$ & $\mathbf{2 5 . 2}$ & $\overline{\mathbf{4 3 . 4}}$ \\
\hline & \multicolumn{6}{|c|}{ Test s1 (seen) } \\
\hline TSN Fusion [14] & 48.2 & 84.1 & 36.7 & 62.3 & 20.5 & 39.8 \\
\hline LFB Max [90] & 60.0 & 88.4 & 45.0 & 71.8 & 32.7 & 55.3 \\
\hline WeakLargeScale [24] & 65.2 & 87.4 & 45.1 & 67.8 & 34.5 & 53.8 \\
\hline$\overline{\mathrm{D}} \overline{\mathrm{E}} \overline{\mathrm{EP}}-\overline{\mathrm{H}} \overline{\mathrm{AL}} \overline{\mathrm{O}} \overline{\mathrm{O}} \overline{\mathrm{D}} \overline{\mathrm{F}}+\overline{\mathrm{S}} \overline{\mathrm{D}} \overline{\mathrm{F}}(\overline{\mathrm{SK}} \overline{\mathrm{O}} \overline{2} \overline{4})$ & $6 \overline{2} . \overline{2}$ & $-\overline{85.0}$ & $\overline{46.1} 1^{-}$ & $69^{-} 3^{-}$ & $\overline{3} 2.5$ & $\overline{5} \overline{3} . \overline{6}$ \\
\hline AssembleNet++ ODF+SDF(SK1024) & 65.0 & 87.8 & 48.8 & 72.5 & 35.0 & 56.1 \\
\hline \multirow[t]{2}{*}{ 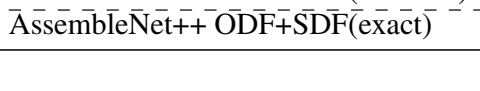 } & $-\overline{6} \overline{2_{2}}-$ & $-\overline{88.5}$ & $\overline{49.3}-$ & $\overline{7} 2.8^{-}$ & $\overline{35.8}$ & $\overline{\mathbf{5}} \overline{\mathbf{6}} . \overline{\mathbf{8}}$ \\
\hline & \multicolumn{6}{|c|}{ Test s2 (unseen) } \\
\hline TSN Fusion [14] & 39.4 & 74.3 & 22.7 & 45.7 & 10.9 & 25.3 \\
\hline LFB Max [90] & 50.9 & 77.6 & 31.5 & 57.8 & 21.2 & 39.4 \\
\hline WeakLargeScale [24] & 57.3 & 81.1 & 35.7 & 58.7 & 25.6 & 42.7 \\
\hline$\overline{\mathrm{DE}} \overline{\mathrm{EP}}-\overline{\mathrm{HAL}} \overline{+} \overline{\mathrm{O}} \overline{\mathrm{D}} \overline{\mathrm{F}}+\overline{\mathrm{S}} \overline{\mathrm{D}} \overline{\mathrm{F}}(\overline{\mathrm{SK}} \overline{\mathrm{O}} \overline{2} \overline{4})$ & $-5 \overline{5} . \overline{3}$ & $-\overline{79 . \overline{1}}$ & $\overline{32.6}$ & $-\overline{55.4}$ & $\overline{2} 2.3^{-}$ & $\overline{3} 9 . \overline{2}$ \\
\hline AssembleNet++ ODF+SDF(SK1024) & 58.3 & 82.1 & 35.2 & 58.2 & 25.9 & 42.9 \\
\hline 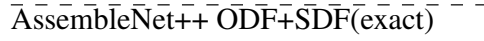 & ${ }^{-} 5 \overline{9} . \overline{0}$ & $-\overline{83 . \overline{3}}$ & $35.7^{-}$ & $59.0^{-}$ & $\overline{27.3}$ & $\overline{44.0}$ \\
\hline
\end{tabular}

Table 8: Experimental results on the EPIC-Kitchens.

supervise the main task. We are the first to hallucinate object and saliency detection descriptors with clear cut improvements in accuracy, and state-of-the-art results on the large-scale Charades and EPIC-Kitchens. More importantly, we demonstrate that hallucinating object and saliency detections is an attractive proposition even for the state-of-the-art AR backbones such as AssembleNet and AssembleNet++.

\section{A Reweighting mechanism}

In this experiment, we employ pipeline ( $D E E P-H A L+W+G+S D F+O D F(S K 512))$ explained above. Typically, we use three levels of weighting mean pooling which are applied to (i) four object detectors constituting on ODF, (ii) two saliency detectors constituting on SDF, and (iii) the final combination of $\mathrm{HAF} / \mathrm{BOW} / \mathrm{FV} / \mathrm{OFF} / \mathrm{ODF} / \mathrm{SDF}$. Thus, below we investigate the performance of a single weighting mean pooling step applied simultaneously to four object detectors, two saliency detectors and the remaining streams. 


\begin{tabular}{lcccc}
\hline & sp1 & sp2 & sp3 & mean acc. \\
\hline wei+flat & $86.47 \%$ & $85.56 \%$ & $86.27 \%$ & $86.10 \%$ \\
wei+3 levels & $88.37 \%$ & $86.80 \%$ & $87.52 \%$ & $\mathbf{8 7 . 5 6 \%}$
\end{tabular}

Table 9: Evaluations of the flat single level weighted mean (wei+flat) vs. three levels of weighted mean pooling (wei+3 levels) on HMDB-51.

\begin{tabular}{|c|c|c|c|c|c|}
\hline & $\begin{array}{l}\text { no. of } \\
\text { frames }\end{array}$ & $\begin{array}{l}\text { av. frame } \\
\text { count }\end{array}$ & $\begin{array}{l}\text { no. of } \\
\text { videos }\end{array}$ & $\begin{array}{l}\text { no. of } \\
\text { clips }\end{array}$ & $\begin{array}{l}\text { no. of } \\
\text { classes }\end{array}$ \\
\hline HMDB-51 & 628635 & 92.91 & 6766 & 6766 & 51 \\
\hline YUP++ & 166463 & 138.72 & 1200 & 1200 & 20 \\
\hline MPII & 662394 & 176.73 & 44 & 3748 & 60 \\
\hline Charades & 19978821 & 300.51 & 9848 & 66500 & 157 \\
\hline
\end{tabular}

Table 10: Statistics of datasets used in our experiemnts.

Table 9 shows that using a flat single level weighted mean pooling yields $86.1 \%$ accuracy on the HMDB-51 which is a $\sim 1.4 \%$ less compared to utilizing three levels of weighted mean pooling. We expect that having one weighted mean pooling per modality is a reasonable strategy as for instance object category detectors may yield similar responses thus they should be first reweighted for the best 'combined detector' performance before being combined with highly complementary modalities.

Finally, Figure 8 (top) demonstrates how our Golden-search selects optimal $\beta$ on the validation set of MPII (split1). Figure 8 (bottom) demonstrates the corresponding validation mAP (this is not the mAP score on the testing set). Note that for the first 10 epochs we use $\beta=0$ and we start the Golden-search from epoch 11.

\section{B Dataset statistics and timing}

Table 10 shows basic statistics re. datasets used in our experiments. We note that Charades with 66500 uniquely annotated clips, 157 action labels and an average frame count of 300 per clip is the largest among these datasets.

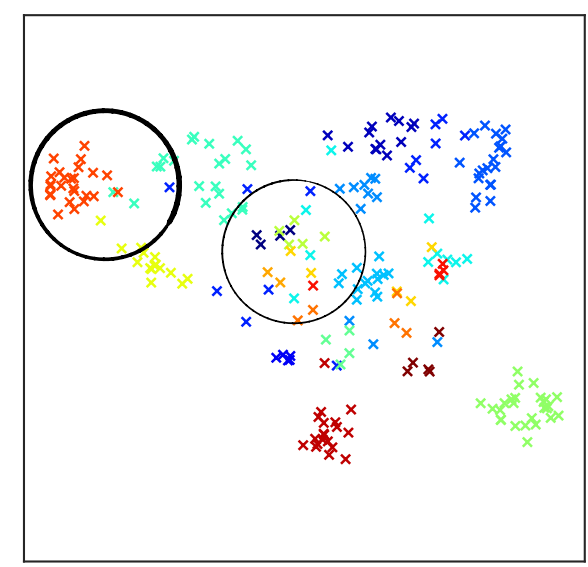

(a)

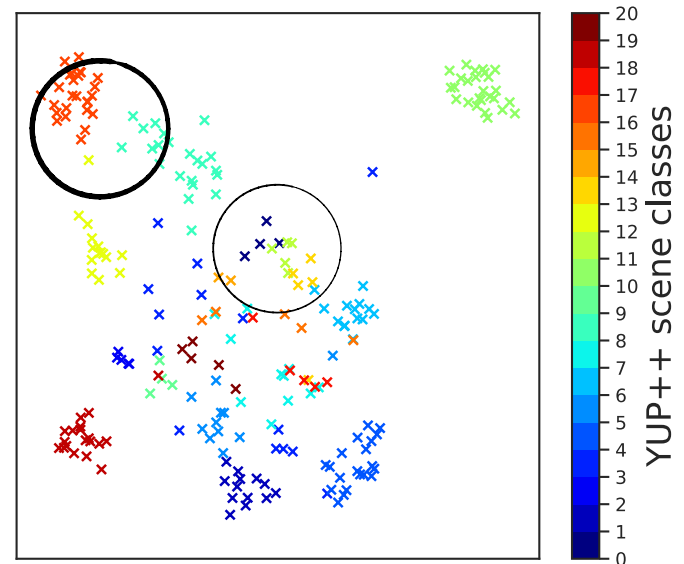

(b)

Figure 6: Visualization of the feature space (from PredNet) for DEEP-HAL in Fig. 6a and DEEP-HAL+ODF in Fig. $6 b$ on the YUP++ dataset. For comparison, we circle regions with interesting changes.

Table 11 introduces timing for object detectors used by our ODF descriptors during training. We note that detections with all four object detectors which we use take $\sim 1.47$ second per frame. Thus, obtaining four ODF descriptors per clip (uniquely annotated sequence to train or classify) takes between 136 and 441 seconds. Table 12 introduces timing for saliency detectors used in our SDF descriptors during training. We note that detections with both saliency detectors which we use take $\sim 0.9$ second per frame, and obtaining both SDF descriptors per clip takes between 84 and 271 seconds. We do note that the major computational cost is incurred due to detectors rather than our ODF and SDF descriptors proposed in the main paper (their cost is minimal). We further note that 


\begin{tabular}{|c|c|c|c|c|c|}
\hline & $\begin{array}{c}\text { DET1: } \\
\text { Inception } \\
\text { V2 }\end{array}$ & $\begin{array}{c}\text { DET2: } \\
\text { Inception } \\
\text { ResNet V2 }\end{array}$ & $\begin{array}{c}\text { DET3: } \\
\text { ResNet101 } \\
\text { AVA }\end{array}$ & $\begin{array}{l}\text { DET4: } \\
\text { NASNet }\end{array}$ & $\begin{array}{c}\text { ODF } \\
\text { total } \\
\text { (+SVD) }\end{array}$ \\
\hline sec. per frame & 0.07 & 0.38 & 0.10 & 0.91 & $1.46(+0.09)$ \\
\hline s.p.c. HMDB-51 & 6.5 & 35.3 & 9.3 & 84.5 & $135.6(+0.5)$ \\
\hline s.p.c. YUP++ & 9.7 & 52.7 & 13.9 & 126.2 & $202.5(+0.8)$ \\
\hline s.p.c. MPII & 12.4 & 67.1 & 17.7 & 160.8 & $258.0(+1.3)$ \\
\hline s.p.c. Charades & 21.0 & 114.2 & 30.0 & 273.5 & $438.7(+2.6)$ \\
\hline
\end{tabular}

Table 11: Statistics of object detectors we use. We provide timings such as seconds per frame (sec. per frame) and seconds per clip (s.p.c.) for detectors used by ODF. The total time incurred by a combined detector $(O D F$ total) is also provided. We also compute the time taken by the full SVD and all remaining ODF operations described in the main paper, assuming $\sim 5$ detections per frame.

\begin{tabular}{lcccc}
\hline & $\begin{array}{c}\text { SAL1: } \\
\text { MNL }\end{array}$ & $\begin{array}{c}\text { SAL2: } \\
\text { ACLNet }\end{array}$ & $\begin{array}{c}\text { SDF } \\
\text { total } \\
\text { (+Eq. (10)) }\end{array}$ & $\begin{array}{c}\text { ODF+SDF } \\
\text { total } \\
\text { (+Eq. (10)+SVD) }\end{array}$ \\
\hline sec. per frame & 0.60 & 0.30 & $0.90(+0.003)$ & $2.36(+0.1)$ \\
\hline s.p.c. HMDB-51 & 55.7 & 27.9 & $83.6(+0.3)$ & $219.2(+0.8)$ \\
s.p.c. YUP++ & 83.2 & 41.6 & $124.8(+0.4)$ & $327.3(+1.2)$ \\
s.p.c. MPII & 106.0 & 53.0 & $159.0(+0.5)$ & $417.0(+1.8)$ \\
s.p.c. Charades & 180.3 & 90.1 & $270.4(+0.9)$ & $709.1(+3.5)$ \\
\hline
\end{tabular}

Table 12: Statistics of saliency detectors we use. We provide timings such as seconds per frame (sec. perframe) and seconds per clip (s.p.c.) for detectors used by SDF. The total time incurred by a combined detector (SDF total) is also provided. We also compute the time taken by the descriptor in Eq. (10) and all remaining SDF operations described in the main paper. Finally, we also provide the combined ODF and SDF time ( $S D F+O D F$ total).

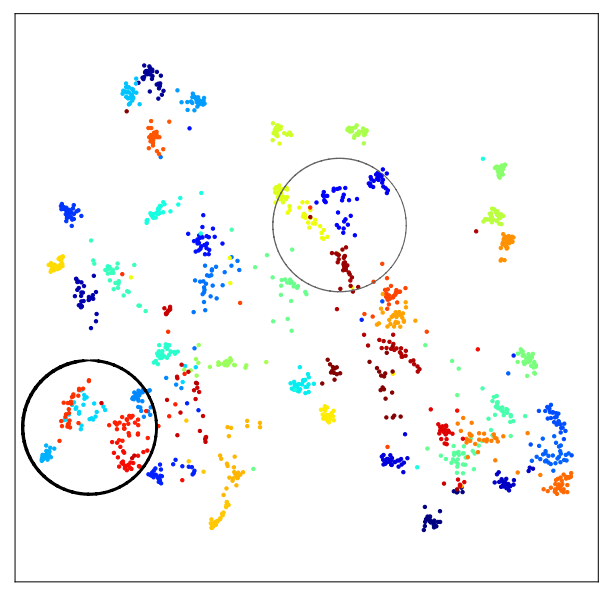

(a)

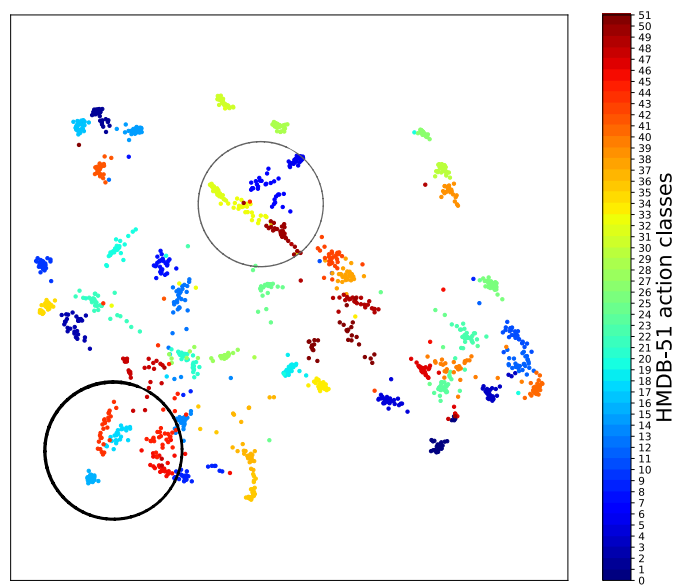

(b)

Figure 7: Visualization of the feature space (from PredNet) for DEEP-HAL in Fig. 7a and DEEP-HAL+ODF in Fig. $7 \mathrm{~b}$ on the HMDB-51 dataset. For comparison, we circle regions with interesting changes.

the idea of learning these costly representations during training is very valuable. While the total computations per training clip vary between 220 and 712 seconds, during testing time we obtain these representations for free (milliseconds) thanks to DET1,..,DET4 and SAL1/SAL2 units from Figure 2 (the main submission). Assuming 25\% of clips in charades for testing, that results in 137 days of computational savings on a single GPU (conversely, 1 day savings on 137 GPUs). Given the obtained $6 \%$ boost on Charades over the baseline without ODF and SDF, and the computational savings, we believe these statistics highlight the value of our approach.

\section{Visualization using UMAP}

Figure 6 is a visualization performed with UMAP [55] on the YUP++ dataset. In Fig. 6a, top left corner contains samples from classes in red, green, and blue colors which partially overlap. In Fig. 

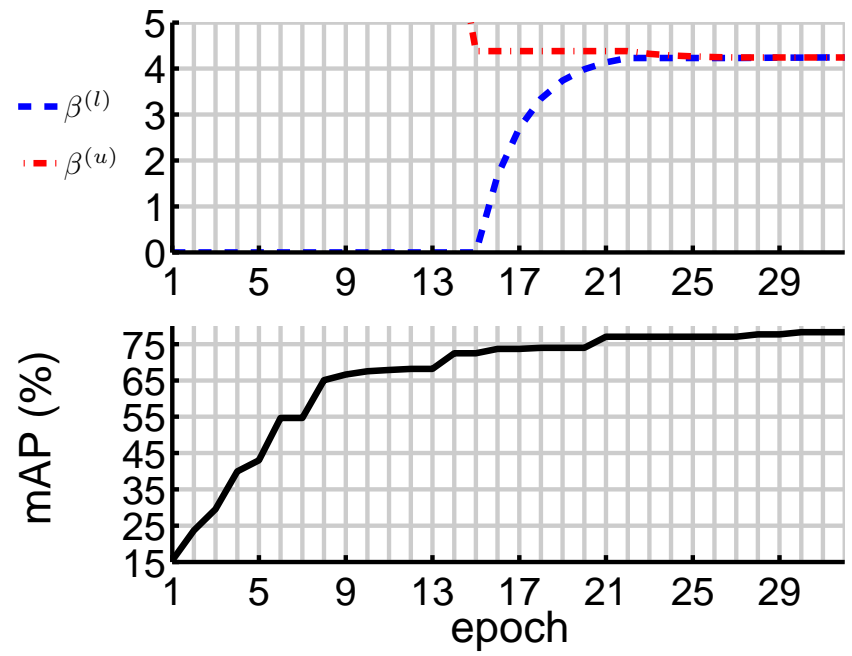

Figure 8: Visualization of the Golden-search for the weighting mechanism (final level weighting). (top) Illustration of how the lower and upper estimates $\beta^{(l)}$ and $\beta^{(u)}$ converge as epochs progress. (bottom) For every epoch, we set $\beta=0.5\left(\beta^{(l)}+\beta^{(u)}\right)$ and obtain the corresponding validation score (mAP) on MPII (split1). As the epoch number advances, mAP improves and remains stable as the Golden-search algorithm converges.

$6 \mathrm{~b}$, top left corner contains the samples from the corresponding classes in red, green, and blue colors. This time, the samples of these three classes are well separated from each other.

Figure 7 is a visualization performed with UMAP [55] on the HMDB-51 dataset. In Fig. 7a, bottom left corner contains samples from classes in red and blue colors which partially overlap. In Fig. 7b, bottom left corner contains the samples from the corresponding classes in red and blue colors. This time, the class-wise clusters seem to be more clearly delineated and samples of these classes are separated better from each other.

\section{References}

[1] Fabien Baradel, Natalia Neverova, Christian Wolf, Julien Mille, and Greg Mori. Object level visual reasoning in videos. In ECCV, pages 1-16, Munich, Germany, September 2018. Springer Science+Business Media. 9

[2] Ali Borji, Ming-Ming Cheng, Huaizu Jiang, and Jia Li. Salient object detection: A benchmark. TIP, 24(12):5706-5722, 2015. 4

[3] Jim Braux-Zin, Romain Dupont, and Adrien Bartoli. A general dense image matching framework combining direct and feature-based costs. In ICCV, pages 185-192, Sydney, NSW, Australia, 2013. IEEE. 4

[4] Thomas Brox and Jitendra Malik. Large displacement optical flow: Descriptor matching in variational motion estimation. TPAMI, 33(3):500-513, March 2011. 4

[5] João Carreira and Andrew Zisserman. Quo Vadis, Action Recognition? A New Model and the Kinetics Dataset. In $C V P R$, pages 1-10, Honolulu, HI, USA, 2018. IEEE. 1, 4, 11

[6] Bhaskar Chakraborty, Michael B. Holte, Thomas B. Moeslund, and Jordi Gonzàlez. Selective spatio-temporal interest points. CVIU, 116(3):396-410, 2012. 3

[7] Anoop Cherian, Basura Fernando, Mehrtash Harandi, and Stephen Gould. Generalized rank pooling for action recognition. In CVPR, pages 3222-3231, Honolulu, HI, USA, 2017. IEEE. 9, 12

[8] Anoop Cherian, Piotr Koniusz, and Stephen Gould. Higher-order pooling of CNN features via kernel linearization for action recognition. In WACV, pages 130-138, Santa Rosa, CA, USA, 2017. IEEE. 1, 3, 4

[9] Anoop Cherian, Suvrit Sra, Stephen Gould, and Richard Hartley. Non-linear temporal subspace representations for activity recognition. In CVPR, pages 2197-2206, Salt Lake City, UT, USA, 2018. IEEE. 1, 3, 4, 9, 12 
[10] Vasileios Choutas, Philippe Weinzaepfel, Jérôme Revaud, and Cordelia Schmid. PoTion: Pose motion representation for action recognition. In CVPR, pages 7024-7033, Salt Lake City, UT, USA, 2018. IEEE. 1, 3

[11] Graham Cormode and Marios Hadjieleftheriou. Finding frequent items in data streams. Proc. VLDB Endow., 1(2):1530-1541, August 2008. 5

[12] Gabriella Csurka, Christopher R. Dance, Lixin Fan, Jutta Willamowski, and Cédric Bray. Visual categorization with bags of keypoints. In ECCV Workshop, pages 1-22, Prague, Czech Republic, 2004. Springer Science+Business Media. 1, 3

[13] Navneet Dalal, Bill Triggs, and Cordelia Schmid. Human Detection Using Oriented Histogram of Flow and Appearance. In ECCV, pages 428-441, Graz, Austria, 2006. Springer Science+Business Media. 1, 3

[14] Dima Damen, Hazel Doughty, Giovanni Maria Farinella, Sanja Fidler, Antonino Furnari, Evangelos Kazakos, Davide Moltisanti, Jonathan Munro, Toby Perrett, Will Price, and Michael Wray. Scaling egocentric vision: The epic-kitchens dataset. In ECCV, pages 1-17, Munich, Germany, September 2018. Springer Science+Business Media. 9, 13

[15] Piotr Dollár, Vincent Rabaud, Garrison Cottrell, and Serge Belongie. Behavior recognition via sparse spatio-temporal features. In ICCCN, pages 65-72, Honolulu, HI, USA, 2005. IEEE. 3

[16] Jeff Donahue, Lisa Anne Hendricks, Sergio Guadarrama, Marcus Rohrbach, Subhashini Venugopalan, Trevor Darrell, and Kate Saenko. Long-term recurrent convolutional networks for visual recognition and description. In CVPR, pages 2625-2634, Boston, MA, USA, 2015. IEEE. 4

[17] Pengfei Fang, Jieming Zhou, Soumava Kumar Roy, Lars Petersson, and Mehrtash Harandi. Bilinear attention networks for person retrieval. In ICCV, pages 8030-8039, Seoul, Korea, 2019. IEEE. 4

[18] Christoph Feichtenhofer, Haoqi Fan, Jitendra Malik, and Kaiming He. Slowfast networks for video recognition. In ICCV, pages 6202-6211, Seoul, Korea, 2019. IEEE. 12

[19] Christoph Feichtenhofer, Axel Pinz, and Richard P. Wildes. Spatiotemporal residual networks for video action recognition. In NIPS, pages 3468-3476, Barcelona, Spain, 2016. MIT Press. 1,4

[20] Christoph Feichtenhofer, Axel Pinz, and Richard P. Wildes. Temporal residual networks for dynamic scene recognition. In CVPR, pages 4728-4737, Honolulu, HI, USA, 2017. IEEE. 9, 11

[21] Basura Fernando, Efstratios Gavves, José Oramas M., Amir Ghodrati, and Tinne Tuytelaars. Modeling video evolution for action recognition. In CVPR, pages 5378-5387, Boston, MA, USA, 2015. IEEE. 4

[22] Basura Fernando and Stephen Gould. Learning end-to-end video classification with rankpooling. In ICML, volume 48, pages 1187-1196, New York City, NY, USA, 2016. ACM. 1, 3, 4

[23] William T. Freeman and Michal Roth. Orientation histograms for hand gesture recognition. Technical Report TR94-03, MERL - Mitsubishi Electric Research Laboratories, Cambridge, MA 02139, December 1994. 3

[24] Deepti Ghadiyaram, Du Tran, and Dhruv Mahajan. Large-scale weakly-supervised pre-training for video action recognition. In CVPR, pages 12046-12055, Long Beach,California,USA, June 2019. IEEE. 13

[25] Ross Girshick. Fast r-cnn. In ICCV, pages 1440-1448, Santiago, Chile, 2015. IEEE. 4

[26] Ross Girshick, Jeff Donahue, Trevor Darrell, and Jitendra Malik. Region-based convolutional networks for accurate object detection and segmentation. TPAMI, 38(1):142-158, January 2016. 4

[27] Chunhui Gu, Chen Sun, David A. Ross, Carl Vondrick, Caroline Pantofaru, Yeqing Li, Sudheendra Vijayanarasimhan, George Toderici, Susanna Ricco, Rahul Sukthankar, Cordelia Schmid, and Jitendra Malik. Ava: A video dataset of spatio-temporally localized atomic visual actions. In CVPR, pages 6047-6056, Salt Lake City, UT, USA, June 2018. IEEE. 2, 4 
[28] Isma Hadji and Richard P. Wildes. A new large scale dynamic texture dataset with application to ConvNet understanding. In ECCV, Munich, Germany, September 2018. Springer Science+Business Media. 11

[29] Kaiming He, Georgia Gkioxari, Piotr Dollár, and Ross B. Girshick. Mask r-cnn. In ICCV, pages 2980-2988, Venice, Italy, 2017. IEEE. 4

[30] Kaiming He, Xiangyu Zhang, Shaoqing Ren, and Jian Sun. Deep residual learning for image recognition. In $C V P R$, pages 1-12, Las Vegas, NV, USA, June 2016. IEEE. 2, 4

[31] Berthold K. P. Horn and Brian G. Schunck. Determining optical flow. Artificial Intelligence, 17:185-203, 1981. 4

[32] Qibin Hou, Ming-Ming Cheng, Xiaowei Hu, Ali Borji, Zhuowen Tu, and Philip H. S. Torr. Deeply supervised salient object detection with short connections. In CVPR, pages 32033212, Honolulu, HI, USA, July 2017. IEEE. 4

[33] Tony Jebara, Risi Kondor, and Andrew Howard. Probability product kernels. JMLR, 5:819$844,2004.5$

[34] Hervé Jégou, Matthijs Douze, and Cordelia Schmid. On the Burstiness of Visual Elements. In CVPR, pages 1169-1176, Long Beach,alifornia, USA, 2009. IEEE. 4

[35] Shuiwang Ji, Wei Xu, Ming Yang, and Kai Yu. 3D convolutional neural networks for human action recognition. TPAMI, 35:221-231, 2010. 4

[36] Andrej Karpathy, George Toderici, Sanketh Shetty, Thomas Leung, Rahul Sukthankar, and Li Fei-Fei. Large-scale video classification with convolutional neural networks. In CVPR, pages 1725-1732, Columbus, OH, USA, 2014. IEEE. 4

[37] Alexander Kläser, Marcin Marszalek, and Cordelia Schmid. A Spatio-Temporal Descriptor Based on 3D-Gradients. In BMCV, pages 1-10, Leeds, UK, 2008. BMVA. 1, 3

[38] Piotr Koniusz, Anoop Cherian, and Fatih Porikli. Tensor representations via kernel linearization for action recognition from 3D skeletons. In ECCV, pages 1-14, Amsterdam, The Netherlands, 2016. Springer Science+Business Media. 4

[39] Piotr Koniusz and Krystian Mikolajczyk. Soft Assignment of Visual Words as Linear Coordinate Coding and Optimisation of its Reconstruction Error. In ICIP, pages 2461-2464, Brussels, Belgium, 2011. IEEE. 3

[40] Piotr Koniusz, Lei Wang, and Anoop Cherian. Tensor representations for action recognition. In IEEE Transactions on Pattern Analysis and Machine Intelligence. IEEE, 2020. 4

[41] Piotr Koniusz, Fei Yan, Philippe-Henri Gosselin, and Krystian Mikolajczyk. Higher-order Occurrence Pooling on Mid- and Low-level Features: Visual Concept Detection. Technical Report, 1(1):1-20, 2013. 4

[42] Piotr Koniusz, Fei Yan, Philippe-Henri Gosselin, and Krystian Mikolajczyk. Higher-order occurrence pooling for bags-of-words: Visual concept detection. TPAMI, 39(2):313-326, 2017. 4

[43] Piotr Koniusz, Fei Yan, and Krystian Mikolajczyk. Comparison of Mid-Level Feature Coding Approaches And Pooling Strategies in Visual Concept Detection. CVIU, 117:479-492, 2012. 3, 4, 5

[44] Piotr Koniusz and Hongguang Zhang. Power normalizations in fine-grained image, few-shot image and graph classification. In IEEE Transactions on Pattern Analysis and Machine Intelligence. IEEE, 2020. 4

[45] Piotr Koniusz, Hongguang Zhang, and Fatih Porikli. A deeper look at power normalizations. In CVPR, pages 5774-5783, Salt Lake City, UT, USA, 2018. IEEE. 4, 5

[46] Hildegard Kuehne, Hueihan Jhuang, Estíbaliz Garrote, Tomaso Poggio, and Thomas Serre. HMDB: A large video database for human motion recognition. In ICCV, pages 2556-2563, Barcelona, Spain, 2011. IEEE. 9

[47] Deepak Kumar, Chetan Kumar, Chun-Wei Seah, Siyu Xia, and Ming Shao. Finding achilles' heel: Adversarial attack on multi-modal action recognition. In Chang Wen Chen, Rita Cucchiara, Xian-Sheng Hua, Guo-Jun Qi, Elisa Ricci, Zhengyou Zhang, and Roger Zimmermann, editors, $M M$, pages 3829-3837, Seattle, United States, 2020. ACM. 4 
[48] Ivan Laptev. On space-time interest points. IJCV, 64(2-3):107-123, September 2005. 3

[49] Chuanzhen Li, Bailiang Su, Jingling Wang, and Qin Zhang. Human action recognition using multi-velocity STIPs and motion energy orientation histogram. J. Inf. Sci. Eng., 30:295-312, 2014. 3

[50] Jiapeng Li, Ping Wei, Yongchi Zhang, and Nanning Zheng. A slow-i-fast-p architecture for compressed video action recognition. In MM, pages 2039-2047, Seattle, United States, 2020. ACM. 4

[51] Tsung-Yi Lin, Michael Maire, Serge Belongie, James Hays, Pietro Perona, Deva Ramanan, Piotr Dollár, and C. Lawrence Zitnick. Microsoft coco: Common objects in context. In David Fleet, Tomas Pajdla, Bernt Schiele, and Tinne Tuytelaars, editors, Computer Vision - ECCV 2014, pages 740-755, Cham, 2014. Springer International Publishing. 2, 4

[52] Liu Lingqiao, Lei Wang, and Xinwang Liu. In Defence of Soft-assignment Coding. In ICCV, pages 2486-2493, Barcelona, Spain, 2011. IEEE. 3

[53] Ziming Liu, Guangyu Gao, A. Kai Qin, Tong Wu, and Chi Harold Liu. Action recognition with bootstrapping based long-range temporal context attention. In Laurent Amsaleg, Benoit Huet, Martha A. Larson, Guillaume Gravier, Hayley Hung, Chong-Wah Ngo, and Wei Tsang Ooi, editors, MM, pages 583-591, Nice, France, 2019. ACM. 4

[54] Julien Mairal, Piotr Koniusz, Zaid Harchaoui, and Cordelia Schmid. Convolutional kernel networks. In NIPS, pages 1-9, Montreal, Quebec, Canada, 2014. MIT Press. 7

[55] Leland McInnes, John Healy, Nathaniel Saul, and Lukas Grossberger. Umap: Uniform manifold approximation and projection. The Journal of Open Source Software, 3(29):861, 2018. 15,16

[56] Nils Papenberg, Andrés Bruhn, Thomas Brox, Stephan Didas, and Joachim Weickert. Highly accurate optic flow computation with theoretically justified warping. IJCV, 67:141-158, 2006. 4

[57] Florent Perronnin and Christopher Dance. Fisher kernels on visual vocabularies for image categorization. In $C V P R$, volume 0, pages 1-8, Minneapolis, Minnesota, USA, 2007. IEEE. 1, 3

[58] Florent Perronnin, Jorge Sánchez, and Thomas Mensink. Improving the Fisher Kernel for Large-Scale Image Classification. In ECCV, pages 143-156, Heraklion, Crete, 2010. Springer Science+Business Media. 1, 3

[59] Ninh Pham and Rasmus Pagh. Fast and scalable polynomial kernels via explicit feature maps. In ACM SIGKDD, pages 239-247, Chicago, USA, 2013. ACM. 5

[60] AJ Piergiovanni, Anelia Angelova, Alexander Toshev, and Michael S. Ryoo. Evolving spacetime neural architectures for videos. In ICCV, pages 1793-1802, Seoul, Korea, October 2019. IEEE. 11

[61] Joseph Redmon, Santosh Divvala, Ross Girshick, and Ali Farhadi. You only look once: Unified, real-time object detection. In CVPR, pages 779-788, Boston, MA, USA, 2015. IEEE. 4

[62] Shaoqing Ren, Kaiming He, Ross Girshick, and Jian Sun. Faster R-CNN: Towards real-time object detection with region proposal networks. In NIPS, pages 91-99, Montreal, Canada, 2015. MIT Press. 2, 4, 9

[63] Jérôme Revaud, Philippe Weinzaepfel, Zaid Harchaoui, and Cordelia Schmid. EpicFlow: Edge-Preserving Interpolation of Correspondences for Optical Flow. In CVPR, pages 11641172, Boston, MA, USA, 2015. IEEE. 4

[64] Marcus Rohrbach, Sikandar Amin, Mykhaylo Andriluka, and Bernt Schiele. A database for fine grained activity detection of cooking activities. In CVPR, pages 1194-1201, Providence, Rhode Island, 2012. IEEE. 9

[65] Olga Russakovsky, Jia Deng, Hao Su, Jonathan Krause, Sanjeev Satheesh, Sean Ma, Zhiheng Huang, Andrej Karpathy, Aditya Khosla, Michael Bernstein, Alexander C. Berg, and Li FeiFei. ImageNet large scale visual recognition challenge. IJCV, 115(3):211-252, 2015. 3, 4

[66] Michael S. Ryoo, AJ Piergiovanni, Juhana Kangaspunta, and Anelia Angelova. Assemblenet++: Assembling modality representations via attention connections. In ECCV, pages 1-19, Glasgow, UK, 2020. Springer Science+Business Media. 4 
[67] Michael S. Ryoo, AJ Piergiovanni, Mingxing Tan, and Anelia Angelova. Assemblenet: Searching for multi-stream neural connectivity in video architectures. In ICLR, pages 1-15, Addis Ababa, Ethiopia, 2020. ICLR. 1, 4, 12

[68] Paul Scovanner, Saad Ali, and Mubarak Shah. A 3-Dimentional SIFT Descriptor and its Application to Action Recognition. In MM, pages 357-356, Augsburg, Germany, 2007. ACM. 1, 3

[69] Gunnar A. Sigurdsson, Gül Varol, Xiaolong Wang, Ali Farhadi, Ivan Laptev, and Abhinav Gupta. Hollywood in homes: Crowdsourcing data collection for activity understanding. In ECCV, pages 1-17, Amsterdam, The Netherlands, 2016. Springer Science+Business Media. 9

[70] Karen Simonyan and Andrew Zisserman. Two-stream convolutional networks for action recognition in videos. In NIPS, pages 568-576, Montreal, Quebec, Canada, 2014. MIT Press. 1, 4

[71] Josef Sivic and Andrew Zisserman. Video Google: A text retrieval approach to object matching in videos. ICCV, 2:1470-1477, 2003. 1, 3

[72] Christian Szegedy, Sergey Ioffe, Vincent Vanhoucke, and Alexander A. Alemi. Inception-v4, inception-resnet and the impact of residual connections on learning. In $A A A I$, pages 42784284, San Francisco,CA,USA, 2017. AAAI Press. 2, 4

[73] Christian Szegedy, Vincent Vanhoucke, Sergey Ioffe, Jon Shlens, and Zbigniew Wojna. Rethinking the inception architecture for computer vision. In CVPR, pages 2818-2826, Las Vegas, NV, USA, June 2016. IEEE. 2, 4

[74] Yongyi Tang, Lin Ma, and Lianqiang Zhou. Hallucinating optical flow features for video classification. In IJCAI, pages 926-932, Macao, China, 2019. IJCAI. 1

[75] Du Tran, Lubomir Bourdev, Rob Fergus, Lorenzo Torresani, and Manohar Paluri. Learning Spatiotemporal Features with 3D Convolutional Networks. In ICCV, pages 4489-4497, Santiago, Chile, 2015. IEEE. 1, 4

[76] Jasper R.R. Uijlings, Ionut Cosmin Duta, Negar Rostamzadeh, and Nicu Sebe. Realtime Video Classification using Dense HOF/HOG. In ICMR, pages 145-152, New York, NY, USA, 2014. ACM. 3

[77] Jan C. van Gemert, Cor J. Veenman, Arnold W. M. Smeulders, and Jan-Mark Geusebroek. Visual word ambiguity. TPAMI, 32(7):1271-1283, July 2010. 3

[78] Gül Varol, Ivan Laptev, and Cordelia Schmid. Long-term temporal convolutions for action recognition. TPAMI, 40(6):1510-1517, 2018. 4

[79] Heng Wang, Alexander Kläser, Cordelia Schmid, and Liu Cheng-Lin. Action Recognition by Dense Trajectories. In CVPR, pages 3169-3176, Colorado Springs, CO, USA, 2011. IEEE. 1, 3

[80] Heng Wang, Alexander Kläser, Cordelia Schmid, and Cheng-Lin Liu. Dense Trajectories and Motion Boundary Descriptors for Action Recognition. IJCV, 103:60-79, 2013. 1, 3

[81] Heng Wang and Cordelia Schmid. Action Recognition with Improved Trajectories. In ICCV, pages 3551-3558, Sydney, Australia, 2013. IEEE. 1, 3

[82] Jue Wang and Anoop Cherian. Learning discriminative video representations using adversarial perturbations. In ECCV, pages 716-733, Munich, Germany, 2018. Springer Science+Business Media. 1, 3, 4, 11

[83] Lei Wang. Analysis and evaluation of Kinect-based action recognition algorithms. Master's thesis, School of the Computer Science and Software Engineering, The University of Western Australia, Nov 2017. 1

[84] Lei Wang, Du Q. Huynh, and Piotr Koniusz. A comparative review of recent kinect-based action recognition algorithms. TIP, 29(1):15-28, 2019. 1

[85] Lei Wang, Piotr Koniusz, and Du Q. Huynh. Hallucinating IDT descriptors and I3D optical flow features for action recognition with cnns. In $I C C V$, pages 8697-8707, Seoul, Korea, October 2019. IEEE. 1, 2, 3, 4, 5, 6, 7, 8, 10, 11, 12, 13

[86] Linzhao Wang, Lijun Wang, Huchuan Lu, Pingping Zhang, and Xiang Ruan. Saliency detection with recurrent fully convolutional networks. In ECCV, pages 825-841, Amsterdam, The Netherlands, 2016. Springer Science+Business Media. 4 
[87] Kilian Weinberger, Anirban Dasgupta, John Langford, Alex Smola, and Josh Attenberg. Feature hashing for large scale multitask learning. In ICML, pages 1113-1120, Montreal, Canada, 2009. ACM. 4, 5

[88] Philippe Weinzaepfel, Jérôme Revaud, Zaid Harchaoui, and Cordelia Schmid. DeepFlow: Large displacement optical flow with deep matching. In ICCV, pages 1-8, Sydney, NSW, Australia, 2013. IEEE. 4

[89] Geert Willems, Tinne Tuytelaars, and Luc Van Gool. An efficient dense and scale-invariant spatio-temporal interest point detector. In ECCV, pages 650-663, Marseille, France, 2008. Springer Science+Business Media. 3

[90] Chao-Yuan Wu, Christoph Feichtenhofer, Haoqi Fan, Kaiming He, Philipp Krahenbuhl, and Ross Girshick. Long-term feature banks for detailed video understanding. In CVPR, pages 284-293, Long Beach, California, USA, June 2019. IEEE. 13

[91] An Yan, Yali Wang, Zhifeng Li, and Yu Qiao. PA3D: Pose-action 3D machine for video recognition. In CVPR, pages 7922-7931, Long Beach, California, USA, June 2019. IEEE. 11

[92] Lahav Yeffet and Lior Wolf. Local trinary patterns for human action recognition. In ICCV, pages 492-497, Seoul, Korea, 2009. IEEE. 3

[93] Can Zhang, Yuexian Zou, Guang Chen, and Lei Gan. PAN: persistent appearance network with an efficient motion cue for fast action recognition. In Laurent Amsaleg, Benoit Huet, Martha A. Larson, Guillaume Gravier, Hayley Hung, Chong-Wah Ngo, and Wei Tsang Ooi, editors, $M M$, pages 500-509, Nice, France, 2019. ACM. 4

[94] Hongguang Zhang, Jing Zhang, and Piotr Koniusz. Few-shot learning via saliency-guided hallucination of samples. In CVPR, pages 2770-2779, Long Beach California, June 2019. IEEE. 3, 4

[95] Jing Zhang, Tong Zhang, Yuchao Dai, Mehrtash Harandi, and Richard Hartley. Deep unsupervised saliency detection: A multiple noisy labeling perspective. In CVPR, pages 1-10, Salt Lake City,UT,USA, June 2018. IEEE. 3, 4

[96] Wangjiang Zhu, Shuang Liang, Yichen Wei, and Jian Sun. Saliency optimization from robust background detection. In CVPR, pages 2814-2821, Columbus, OH, USA, 2014. IEEE. 4

[97] Barret Zoph, Vijay Vasudevan, Jonathon Shlens, and Quoc V. Le. Learning transferable architectures for scalable image recognition. In CVPR, pages 1-14, Salt Lake City, UT, USA, June 2018. IEEE. 2, 4 